\title{
ON THE DEGREE OF APPROXIMATION OF A FUNCTION BY THE PARTIAL SUMS OF ITS FOURIER SERIES $\left(^{1}\right)$ \\ BY

\author{
ELAINE COHEN
}

\begin{abstract}
ABSTRACr. When $f$ is a $2 \pi$ periodic function with $r$ th order fractional derivative, $r>0$, of $p$-bounded variation, Golubov has obtained estimates of the degree of approximation of $f$, in the $L^{q}$ norm, $q>p$, by the partial sums of its Fourier series. Here we consider the analogous problem for functions whose fractional derivatives are of $\Phi$-bounded variation and obtain estimates of the degree of approximation in an Orlicz space norm. In a similar manner we shall extend various results that he obtained on degree of approximation in the sup norm.
\end{abstract}

\section{$\S I$}

Let $f$ be a real function of period $2 \pi$. If the $r$ th fractional derivative, $r \geqslant 0$, is of bounded $p$-variation, Golubov [3] has obtained estimates of the degree of approximation of $f$, in the sup and $L^{q}$ norms, $q \geqslant p$, by the partial sums of the Fourier series of $f$.

We consider the analogous problem for functions whose fractional derivatives are of $\Phi$-bounded variation. In 2.1 we extend Salem's theorem of uniform convergence of Fourier series. In 2.2 we obtain estimates on the degree of approximation in an Orlicz space norm.

In this chapter we shall review the background material which we shall require. In \$1 we concern ourselves with Orlicz spaces, \$2 will present basic information on $\Phi$-bounded variation, and $\$ 3$ contains the relevant information on the fractional integral and derivative.

1. Orlicz spaces. In this section we shall summarize some results on Orlicz spaces which we shall require. Unless otherwise noted, a complete presentation of this topic can be found in the texts of Krasnosel'skii and Rutickii [4] and Zygmund [14].

Let $\varphi$ be a nondecreasing right continuous function, $\varphi: R^{+} \rightarrow R^{+}, \varphi(0)=$

Presented to the Society, January 25, 1975 under the title On the degree of approximation by partial sums of Fourier series in certain Orlicz space norms; received by the editors May 24, 1974 and, in revised form, August 21, 1975.

AMS (MOS) subject classifications (1970). Primary 42A20, 42A16; Secondary 41A25.

Key words and phrases. Orlicz class, Orlicz space, $\Delta_{2}$-condition, $\Delta^{\prime}$-condition, $\Phi$-modulus of continuity, $\Phi$-variation, fractional integral, fractional derivative, degree of approximation.

(') This paper is based on a Ph. D. Dissertation written at Syracuse University under the direction of Professor Daniel Waterman.

- American Mathematical Society 1978 
$0, \lim _{x \rightarrow \infty} \varphi(x)=\infty$. Define

$$
\varphi^{-1}(y)=\sup _{\varphi(x)<y} x .
$$

Then $\varphi^{-1}$ is right continuous, nondecreasing, $\varphi^{-1}: R^{+} \rightarrow R^{+}, \varphi^{-1}(0)=0$, $\lim _{y \rightarrow \infty} \varphi^{-1}(y)=\infty$. If $\varphi$ is strictly increasing then the usual definition of $\varphi^{-1}$ and the above definition coincide.

Let

$$
\Phi(x)=\int_{0}^{|x|} \varphi(t) d t \text { and } \Phi^{*}(x)=\int_{0}^{|x|} \varphi^{-1}(t) d t .
$$

$\Phi$ and $\Phi^{*}$ are both convex, strictly increasing functions such that

$$
\lim _{x \rightarrow 0} \Phi(x) / x=\lim _{y \rightarrow 0} \Phi^{*}(y) / y=0, \quad \lim _{x \rightarrow \infty} \Phi(x) / x=\lim _{y \rightarrow \infty} \Phi^{*}(y) / y=\infty,
$$

and (Young's inequality): for $a, b$ real numbers,

$$
a b \leqslant \Phi(a)+\Phi^{*}(b)
$$

equality occurring only when $b=\varphi(|a|) \operatorname{sgn} a$.

It is known that if $M$ is any convex strictly increasing function such that

$$
\lim _{u \rightarrow 0} M(u) / u=0, \quad \lim _{u \rightarrow \infty} M(u) / u=\infty,
$$

then there exists a right continuous nondecreasing $m(x) ; m: R^{+} \rightarrow R^{+}$, $m(0)=0, \lim _{x \rightarrow \infty} m(x)=\infty$ and

$$
M(x)=\int_{0}^{|x|} m(t) d t
$$

All functions which admit this representation are called Young's functions or $N$-functions.

Henceforth, we consider integrable functions $f$ of period $2 \pi$; integration will be with respect to Lebesgue measure and will extend over a full period unless otherwise noted. Define

$$
L_{\Phi}=\left\{f: \int \Phi(f)<\infty\right\}
$$

and

$$
L_{\Phi}^{*}=\left\{f: k f \in L_{\Phi} \text { for some real number } k \neq 0\right\} .
$$

$L_{\Phi}$ is called an Orlicz class. With norm

$$
\|f\|_{\Phi}=\sup \left\{\int f g: \int \Phi^{*}(g) \leqslant 1\right\}
$$

$L_{\Phi}^{*}$ is a Banach space, and is called an Orlicz space.

Luxemburg introduced an alternative norm on $L_{\Phi}^{*}$ :

$$
\|f\|_{\Phi}^{\prime}=\inf \left\{k: \int \Phi(f / k) \leqslant 1, k \geqslant 0\right\} .
$$


We have the relation $\|\cdot\|_{\Phi}^{\prime} \leqslant\|\cdot\|_{\Phi} \leqslant 2\|\cdot\|_{\Phi}^{\prime}$, making them equivalent norms, but, for $f \neq 0$ a.e., $\|f\|_{\Phi} /\|f\|_{\Phi}^{\prime}=C, C$ not dependent on $f$, if and only if $\Phi(u)=A|u|^{p}, p>1$.

It is known that for all $f \in L_{\Phi}^{*}, g \in L_{\Phi *}^{*}$

$$
\begin{gathered}
\int \Phi(f)<\|f\|_{\Phi}^{\prime} \quad \text { if and only if }\|f\|_{\Phi}^{\prime} \leqslant 1, \\
\|f\|_{\Phi}^{\prime} \leqslant \int \Phi(f) \text { otherwise, }
\end{gathered}
$$

and

$$
\int f g<\left\{\begin{array}{l}
\|f\|_{\Phi}\|g\|_{\Phi^{*}} \\
\|f\|_{\Phi}^{\prime}\|g\|_{\Phi^{*}} \\
\|f\|_{\Phi}\|g\|_{\Phi^{*}}^{\prime}
\end{array}\right.
$$

We introduce the $\Phi$-moduli of continuity of $f$ :

$$
\omega_{\Phi}(\delta ; f)=\sup _{|h|<\delta}\|f(x+h)-f(x)\|_{\Phi}
$$

and

$$
\omega_{\Phi}^{\prime}(\delta ; f)=\sup _{|h|<\delta}\|f(x+h)-f(x)\|_{\Phi}^{\prime} .
$$

Then $\omega_{\Phi}(0 ; f)=\omega_{\Phi}^{\prime}(0 ; f)=0 ; \omega_{\Phi}$ and $\omega_{\Phi}^{\prime}$ are nondecreasing. These functions are continuous if $f$ can be approximated in norm by trigonometric polynomials. The definitions given above are analogous to those of $\omega(p), p$-modulus of continuity of $f \in L^{p}$ and of $\omega$, the modulus of continuity of $f \in C$.

We say that an $N$-function $\Phi$ satisfies the $\Delta_{2}$-condition ("is $\Delta_{2}$ ") if there exists $k>0$ such that for $u>0, \Phi(2 u) \leqslant k \Phi(u)$.

We say that $\Phi$ satisfies the $\Delta_{2}$-condition for large (small) values if there exists $k, u_{0}>0$ such that for $u>u_{0}\left(u \leqslant u_{0}\right), \Phi(2 u)<k \Phi(u)$.

The following conditions are equivalent:

1. $\Phi$ is $\Delta_{2}$ (for large values, for small values).

2. For each $c>1$, there exists $k(c)$ such that $\Phi(c u)<k(c) \Phi(u)$ for all $u>0\left(u>\right.$ some $u_{0}, u$ some $\left.u_{0}\right)$.

3. There exists $\alpha>1$ such that, if $\varphi$ is the right continuous right derivative of $\Phi$,

$$
|u| \varphi(|u|) / \Phi(u)<\alpha
$$

for all $u \neq 0$ (for $|u| \geqslant$ some $u_{0}$, for $0<|u|<$ some $u_{0}$ ).

From condition 3 we have that if $\Phi$ is $\Delta_{2}$ for large values, $|u|>u_{0}$, then

$$
\Phi(u)<\Phi\left(u_{0}\right)|u|^{\alpha} / u_{0}^{\alpha} ;
$$

if $\Phi$ is $\Delta_{2}$ for small values, $|u| \leqslant u_{0}$, then 


$$
\Phi(u) \geqslant \Phi\left(u_{0}\right)|u|^{\alpha} / u_{0}^{\alpha} ;
$$

and if $\Phi$ is $\Delta_{2}$, both these inequalities hold.

We have, further, that $L_{\Phi}^{*}=L_{\Phi}$ if and only if $\Phi$ is $\Delta_{2}$ for large values, and $L_{\Phi}^{*}$ is separable if and only if $\Phi$ is $\Delta_{2}$ for large values. When $L_{\Phi}^{*}$ is separable, the polynomials are dense in $L_{\Phi}^{*}$. Thus, we see that we can guarantee continuity of $\omega_{\Phi}$ if $\Phi$ is $\Delta_{2}$ for large values; otherwise, continuity depends on the function $f \in L_{\Phi}^{*}$.

If we have a sequence $\left\{f_{n}\right\}_{n=1}^{\infty} \in L_{\Phi}^{*}$ then norm convergence to, say, $f_{0}$ implies mean convergence to $f_{0} \in L_{\Phi}^{*}$. The converse is true if and only if $\Phi$ is $\Delta_{2}$ for large values.

We define a partial ordering on the set of $N$-functions by $\Phi_{1}<\Phi_{2}$ if there exists $u_{0}, a>0$ such that for $u>u_{0}$,

$$
\Phi_{2}(u) \leqslant a \Phi_{1}(u) .
$$

$\Phi_{1} \sim \Phi_{2}$ (read "is equivalent to") if $\Phi_{1}<\Phi_{2}<\Phi_{1}$. It is known that $\Phi_{1}<\Phi_{2}$ implies that $L_{\Phi_{1}} \subseteq L_{\Phi_{2}}$, and, hence, that equivalent $N$-functions generate the same Orlicz class. Clearly, then, if $\Phi_{1} \sim \Phi_{2}$ and $\Phi_{1}$ is $\Delta_{2}$ for large values, then $\Phi_{2}$ is also. It is known that in each equivalence class of functions which are $\Delta_{2}$ for large values there is at least one function which is $\Delta_{2}$.

From the remarks following condition 3 we have that " $\Phi$ is $\Delta_{2}$ for large values" implies that

$$
L^{\alpha}=\left\{f: \int|f|^{\alpha}<\infty\right\} \subseteq L_{\Phi}=L_{\Phi}^{*} .
$$

Ryan [10] has shown that reflexivity of $L_{\Phi}^{*}$ is equivalent to both $\Phi$ and $\Phi^{*}$ satisfying the $\Delta_{2}$-condition for large values.

Orlicz first introduced $\left(L_{\Phi}^{*},\|\cdot\|_{\Phi}\right)$ with the restriction that $\Phi$ be $\Delta_{2}$ for large values; Zygmund removed that restriction.

We say that $\Phi$ satisfies the $\Delta^{\prime}$-condition if there exists $c>0$ such that for $x$, $y>0$,

$$
\Phi(x y)<c \Phi(x) \Phi(y) .
$$

We say that $\Phi$ satisfies the $\Delta^{\prime}$-condition for large (small) values if there exists $c>0$ and $u_{0}>0$ such that for $|x|,|y|>u_{0}\left(|x|,|y|<u_{0}\right)$

$$
\Phi(x y)<c \Phi(x) \Phi(y) .
$$

All functions which are $\Delta^{\prime}$ in one of the above senses are also $\Delta_{2}$ in that sense, but the converse implication is not true.

If $\Phi$ is $\Delta^{\prime}\left(\Delta_{2}\right)$ for small values, say $|u|<u_{0}$, then we can replace $u_{0}$ by any $w>u_{0}$, but the $\Delta^{\prime}\left(\Delta_{2}\right)$ constant increases unboundedly with $w$ unless $\Phi$ is $\Delta^{\prime}$ $\left(\Delta_{2}\right)$ for all values. 


\section{2. $\Phi$-variation.}

We shall require only a few basic notions concerning generalized variation. A more detailed presentation is found in the papers of L. C. Young [13], E. R. Love [5], and Musielak and Orlicz [6].

We define, for an $N$-function $\Phi$,

$$
V_{\Phi}(f ; I)=\sup _{Q} \sum \Phi\left(f\left(x_{i}\right)-f\left(x_{i-1}\right)\right),
$$

where the supremum is taken over all partitions $Q$ of the interval $I$. We say that $f \in \mathfrak{T}_{\Phi}(I)$ if $V_{\Phi}(f ; I)<\infty$.

Define

$$
V_{\Phi}^{(\delta)}(f ; I)=\sup _{|Q|<\delta} \sum \Phi\left(f\left(x_{i}\right)-f\left(x_{i-1}\right)\right)
$$

where $|Q|$ denotes the mesh of the partition $Q$, and let

$$
V_{\Phi}^{*}(f ; I)=\lim _{\delta \rightarrow 0^{+}} V_{\Phi}^{(\delta)}(f ; I) \text {. }
$$

It is easily shown that $f \in \mathfrak{T}_{\Phi}(I)$ if and only if $V_{\Phi}^{*}(f ; I)<\infty$.

We define $f \in \mathfrak{V}_{\Phi}^{*}(I)$ if there exists $k \neq 0$ such that $(k f) \in \mathfrak{V}_{\Phi}(I)$. The class $\mathfrak{V}_{\Phi}^{*}(I)$ is linear, but $\mathfrak{T}_{\Phi}(I)$ is linear (and $=\mathfrak{V}_{\Phi}^{*}(I)$ ) if and only if $\Phi$ satisfies the $\Delta_{2}$-condition for small values.

If the interval with which we are concerned is $[0,2 \pi]$, or any period, we shall omit all reference to it and write $V_{\Phi}(f), \Upsilon^{*} *$, etc.

We define two variational seminorms by

$$
\|f\|_{\Phi V}=\sup \left\{\sum\left(f\left(x_{i}\right)-f\left(x_{i-1}\right)\right) b_{i}: \sum \Phi^{*}\left(b_{i}\right)<1, \text { all } Q\right\},
$$

where the supremum is taken simultaneously over all partitions $Q$ and all sequences $\left\{b_{i}\right\}$ such that $\Sigma \Phi^{*}\left(b_{i}\right) \leqslant 1$, and

$$
\|f\|_{\Phi V}^{\prime}=\inf \left\{k: V_{\Phi}(f / k) \leqslant 1\right\} .
$$

Their equivalence is shown by the relation

$$
\|\cdot\|_{\Phi V}^{\prime} \leqslant\|\cdot\|_{\Phi V} \leqslant 2\|\cdot\|_{\Phi V}^{\prime} \cdot
$$

Another partial ordering is defined on the set of all $N$-functions by $\Phi_{1}(u) \ll \Phi_{2}(u)$ if there exist $a, u_{0}>0$ such that for $|u| \leqslant u_{0}$,

$$
\Phi_{2}(u) \leqslant a \Phi_{1}(u) .
$$

It is known that $\Phi_{1} \ll \Phi_{2}$ implies $\mathfrak{V}_{\Phi_{1}} \subseteq \mathfrak{V}_{\Phi_{2}}$, and thus defines another equivalence relation on the set of $N$-functions.

Functions $f$ of $\Phi$ bounded variation can have simple discontinuities only. We shall assume that they are normalized so that

$$
f(x)=\frac{1}{2}(f(x+)+f(x-)) .
$$


3. Fractional integral and derivative. In addition to our previous assumptions concerning the integrability of $f$, we shall assume that $\int f=0$.

We may choose the constant of integration so that the mean value of $f_{(1)}$, the integral of $f$, is zero. Since $f_{(1)}$ is periodic and of mean value zero, when integrating it we can choose the constant of integration so that $f_{(2)}$ is periodic and has mean value zero. In general, having defined $f_{(1)}, \ldots, f_{(r-1)}$, choose for $f_{(r)}$ that primitive of $f_{(r-1)}$ whose mean value is zero, i.e. if

$$
f(x) \sim S[f]=\sum^{\prime} c_{n} e^{i n x}=\sum_{n \neq 0} c_{n} e^{i n x},
$$

where $S[\cdot]$ represents the Fourier series, then

$$
f_{(r)}(x) \sim \sum^{\prime} c_{n} e^{i n x}(i n)^{-r}=\frac{1}{2 \pi} \int f(t) D^{(r)}(x-t) d t
$$

where

$$
D^{(r)}(t)=\sum^{\prime}(\text { in })^{-r} e^{i n t}
$$

For $0<t<2 \pi$, the function $D^{(r)}(t)$ is a polynomial of degree $r$. We may consider (*) to be a definition of $f_{(r)}$, the fractional integral of order $r$, for all $r>0$, provided we set

$$
(\text { in })^{-r}=|n|^{r} \exp \left(-\frac{1}{2} i \pi r \operatorname{sgn} n\right), \quad n \neq 0 .
$$

The series (*)' can be written

$$
2 \cos \frac{1}{2} \pi r \sum_{n=1}^{\infty} n^{-r} \cos n t+2 \sin \frac{1}{2} \pi r \sum_{n=1}^{\infty} n^{-r} \sin n t .
$$

We know that this converges for $t \neq 0$ to a sum $D^{(r)}(t)$ and that it is $S\left[D^{(r)}\right]$. Thus, the integral in (*) exists almost everywhere and its value, $f_{(r)}(x)$, is integrable. It is known that the series in (*) converges almost everywhere and is $S\left[f_{(r)}\right]$, and $f_{(r)}$ coincides with the ordinary integral for $r=1,2, \ldots$ Thus

$$
D^{(r)}(t)=\sum_{n=1}^{\infty} n^{-r} \cos \left(n t-\frac{1}{2} \pi r\right)
$$

and

$$
\begin{aligned}
f_{(r)}(x)= & \cos \frac{1}{2} \pi r \sum_{n=1}^{\infty} n^{-r}\left(a_{n} \cos n x+b_{n} \sin n x\right) \\
& +\sin \frac{1}{2} \pi r \sum_{n=1}^{\infty} n^{-r}\left(a_{n} \sin n x-b_{n} \cos n x\right) .
\end{aligned}
$$

The fractional integral operator is "additive", i.e. $f_{(\alpha+\beta)}=\left(f_{(\alpha)}\right)_{(\beta)}$, almost everywhere.

Let $r>0$, and $m$ an integer such that $m-1<r<m$. The derivative of $f$ of fractional order $r$ is generally defined by 


$$
f^{(r)}(x)=d^{m} f_{(m-r)}(x) / d x^{m} .
$$

If we suppose $0<r<1$, and $f_{(1-r)}(x)$ is absolutely continuous, then

$$
S\left[f^{(r)}\right]=S^{\prime}\left[f_{(1-r)}\right]=\sum(i n)^{r} c_{n} e^{i n x},
$$

and, almost everywhere,

$$
f(x)=\int D^{(r)}(x-t) f^{(r)}(t) d t .
$$

In other words, $f$ is the fractional integral of order $r$ of $f^{(r)}$. We shall adopt a more restrictive definition so that the fractional derivative always has this property.

We say that $g(x) \in L^{1}(-\pi, \pi)$ is an $r$ th order fractional derivative of $f \in L^{-1}(-\pi, \pi), r>0$, if

(1) $\int f=\int g=0$,

(2) $f(x)=\int D^{(r)}(x-t) g(t) d t$, a.e.

We denote $g$ by $f^{(r)} ; f^{(0)}$ will denote the function $f$. This definition is sufficient for our purposes and will be used throughout.

If we assume that $f$ has an $r$ th derivative, $g$, according to this last definition, then if

$$
g(x) \sim \sum^{\prime} \gamma_{n} e^{i n x}
$$

we have, almost everywhere,

$$
\begin{aligned}
f(x)=g_{(r)}(x) & =\sum^{\prime} \gamma_{n}(i n)^{-r} e^{i n x}=\sum^{\prime} c_{n} e^{i n x}, \\
g(x) & \sim \sum^{\prime}(i n)^{r} c_{n} e^{i n x}
\end{aligned}
$$

and $f_{(m-r)}=g_{(m)}$.

If $f(h ; x)$ denotes the Steklov function,

$$
\frac{1}{h} \int_{-h / 2}^{h / 2} f(x+t) d t
$$

and $0<m-1<r<m$, we have, almost everywhere,

$$
\begin{aligned}
(f(h ; x))^{(r)} & =\frac{1}{h} \frac{d^{m}}{d x^{m}} \int_{-h / 2}^{h / 2} \int D^{(m-r)}(x-t) f(y+t) d t d y \\
& =\frac{1}{h} \frac{d^{m}}{d x^{m}} \int_{-h / 2}^{h / 2} \int D^{(m-r)}((x+y)-t) f(t) d t d y \\
& =\frac{1}{h} \frac{d^{m}}{d x^{m}} \int_{-h / 2}^{h / 2} f_{(m-r)}(x+y) d y \\
& =\frac{1}{h} \int_{-h / 2}^{h / 2} f^{(r)}(x+y) d y=f^{(r)}(h ; x) .
\end{aligned}
$$


The functions we shall consider are in class $L_{\Phi}^{*}$ and have $r$ th fractional derivative in class $\mathfrak{T}_{\Phi}, r \geqslant 0$. Thus, the functions and their $r$ th derivatives have mean value zero. The class $\mho_{\Phi}^{(r)}, r \geqslant 0$, will denote the class of functions $f$ such that $f^{(r)}$ exists, $f^{(r)} \in \mathfrak{T}_{\Phi}$, and,

$$
f^{(r)}(x)=\frac{1}{2}\left(f^{(r)}(x+)+f^{(r)}(x-)\right) \text {. }
$$

$\S$ II

1. Approximation in the $C$-norm. We devote this section to generalizations of Salem's theorem on uniform convergence [11].

1.1. Theorem. If $f^{(r)} \in \Upsilon_{\Phi}^{*}, 0 \leqslant r<\infty, \Sigma \Phi^{*}(1 / k)<\infty, f^{(r)}$ continuous, then $\left\|f-S_{n}(f)\right\|_{C}=o\left(n^{-r}\right)$.

Proof. For $r=0$ the conclusion is Salem's theorem so we assume $r>0$. Define

$$
D_{n}^{(r)}=\sum_{k=n+1}^{\infty} k^{-r} \cos \left(k t-\frac{1}{2} \pi r\right)=D_{0}^{(r)}(t)-S_{n}\left(D_{0}^{(r)} ; t\right)
$$

and

$$
\varphi_{x}(t)=f^{(r)}(x+t)-f^{(r)}(x)
$$

Then

$$
\begin{aligned}
f(x)-S_{n}(f ; x) & =\frac{1}{\pi} \int_{-\pi}^{\pi} D_{n}^{(r)}(t)\left(f^{(r)}(x-t)-f^{(r)}(x)\right) d t \\
& =\frac{1}{\pi}\left(\int_{0<|t|<\pi / n}+\int_{\pi / n<|t|<\pi}\right) D_{n}^{(r)}(t) \varphi_{x}(-t) d t .
\end{aligned}
$$

It is known [9] that

$$
\int_{0<|t|<\pi / n}\left|D_{n}^{(r)}(t)\right| d t=O\left(n^{-r}\right)
$$

hence,

$$
\frac{1}{\pi} \int_{0<|t|<\pi / n} D_{n}^{(r)}(t) \varphi_{x}(-t) d t=o\left(n^{-r}\right),
$$

since $f^{(r)}$ is continuous.

We follow [8] and perform an Abel transformation on the remaining integral, which yields

$$
\begin{aligned}
\frac{1}{\pi} \int_{\pi / n<|t|<\pi} D_{n}^{(r)}(t) \varphi_{x}(-t) d t \\
=\frac{(-1)}{\pi(n+1)^{r}} \int_{\pi / n<|t|<\pi} A_{n}^{(r)}(t) \varphi_{x}(-t) d t \\
\quad+\frac{1}{\pi} \sum_{k=n+1}^{\infty}\left(k^{-r}-(k+1)^{-r}\right) \int_{\pi / n<|t|<\pi} A_{k}^{(r)}(t) \varphi_{x}(-t) d t
\end{aligned}
$$


where

$$
\begin{aligned}
A_{k}^{(r)}(t)= & \sin \left(\frac{2 k+1}{2} t-\frac{1}{2} r \pi\right) / 2 \sin \frac{t}{2} \\
= & \cos \frac{1}{2} r \pi \sin \left(\frac{2 k+1}{2} t\right) / 2 \sin \frac{t}{2} \\
& +\sin \frac{1}{2} r \pi \cos \left(\frac{2 k+1}{2} t\right) / 2 \sin \frac{t}{2} .
\end{aligned}
$$

We want to show that

$$
\int_{\pi / n<|t|<\pi} A_{k}^{(r)}(t) \varphi_{x}(-t) d t=o(1)
$$

uniformly in $x$ as $n \rightarrow \infty(k>n)$. Now let

$$
\int_{\pi / n<|t|<\pi} A_{k}^{(r)}(t) \varphi_{x}(-t) d t=\cos \frac{1}{2} r \pi J_{n}^{(k)}(t)+\sin \frac{1}{2} r \pi H_{n}^{(k)}(t) .
$$

Letting $D_{k}$ denote the $k$ th Dirichlet kernel and observing that $k \geqslant n$,

$$
\begin{aligned}
J_{n}^{(k)}(x) & =\int_{\pi / n<|t|<\pi} D_{k}(t) \varphi_{k}(-t) d t \\
& =\int_{\pi / n<|t|<\pi} \frac{\sin k t}{t} \varphi_{x}(t) d t+o(1)
\end{aligned}
$$

where $o(1)$ is uniform in $x$ as $n \rightarrow \infty$.

Let

$$
J_{n,+}^{(k)}(x)=\int_{\pi / n<|t|<\pi} \frac{\sin k t}{t} \varphi_{x}(t) d t=L_{n}^{(k)}(x)+o(1),
$$

where $o(1)$ is uniform in $x$ as $n \rightarrow \infty$, and

$$
L_{n}^{(k)}(x)=\sum_{j=[k / n]}^{k-1} \int_{j \pi / k}^{(j+1) \pi / k} \frac{\sin k t}{t} \varphi_{x}(t) d t .
$$

Letting $k t=u$, we obtain, for some $\theta \in(\pi, 2 \pi)$,

$$
\begin{aligned}
L_{n}^{(k)}(x) & =\int_{\pi}^{2 \pi} \sum_{j=[k / n]-1}^{k-2}(-1)^{j} \varphi_{x}\left(\frac{u+j \pi}{k}\right) \frac{\sin u}{u+j \pi} d u \\
& =\pi \sin \theta \sum_{j=[k / n]-1}^{k-2}(-1)^{j} \varphi_{x}\left(\frac{\theta+j \pi}{k}\right) \frac{1}{\theta+j \pi} .
\end{aligned}
$$

Without loss of generality, we assume the number of terms in the summation to be even. If the number of terms is odd, we may omit either the first or the last term, both of which are $o(1)$ as $n \rightarrow \infty$.

Neglecting signs, the sum of two consecutive terms is 


$$
\begin{aligned}
\frac{\varphi_{x}\left(\frac{\theta+h \pi}{k}\right)}{\theta+2 h \pi}-\frac{\varphi_{x}\left(\frac{\theta+(2 h+1) \pi}{k}\right)}{\theta+(2 h+1) \pi} & =\frac{\varphi_{x}\left(\frac{\theta+2 h \pi}{k}\right)-\varphi_{x}\left(\frac{\theta+(2 h+1) \pi}{k}\right)}{\theta+2 h \pi} \\
& +\varphi_{x}\left(\frac{\theta+(2 h+1) \pi}{k}\right)\left(\frac{1}{\theta+2 h \pi}-\frac{1}{\theta+(2 h+1) \pi}\right) .
\end{aligned}
$$

Now,

$$
\left|\varphi_{x}\left(\frac{\theta+(2 h+1) \pi}{k}\right)\left(\frac{1}{\theta+2 h \pi}-\frac{1}{\theta+(2 h+1) \pi}\right)\right|<\frac{\omega\left(\frac{(2 h+3) \pi}{k} ; f^{(r)}\right)}{(2 h+1)(2 h+2) \pi} .
$$

Choosing an integer $M, M=M(n) \simeq \vee n$, the sum of the first $M$ of these terms is

$$
<c \omega\left(c M / k+o(1) ; f^{(r)}\right)=c \omega\left(o(1) ; f^{(r)}\right)=o(1)
$$

as $n \rightarrow \infty$. The sum of the remaining terms is

$$
<\frac{c \sup \left|f^{(r)}\right|}{M+[k / n]}=o(1) \quad \text { as } n \rightarrow \infty \text {. }
$$

Considering

$$
\frac{\varphi_{x}\left(\frac{\theta+2 h \pi}{k}\right)-\varphi_{x}\left(\frac{\theta+(2 h+1) \pi}{k}\right)}{\theta+2 h \pi}
$$

and replacing $\theta+2 h \pi$ by $(2 h+2) \pi$ in the denominator, there is an error

$$
\begin{aligned}
& \left|\varphi_{x}\left(\frac{\theta+2 h \pi}{k}\right)-\varphi_{x}\left(\frac{\theta+(2 h+1) \pi}{k}\right)\right|\left|\frac{1}{\theta+2 h \pi}-\frac{1}{(2 h+2) \pi}\right| \\
& \quad=\left|\varphi_{x}\left(\frac{\theta+2 h \pi}{k}\right)-\varphi_{x}\left(\frac{\theta+(2 h+1) \pi}{k}\right)\right|\left|\frac{2 \pi-\theta}{(\theta+2 h \pi)(2 h+2) \pi}\right| \\
& \quad \leqslant\left|f^{(r)}\left(x+\frac{\theta+2 h \pi}{k}\right)-f^{(r)}\left(x+\frac{\theta+(2 h+1) \pi}{k}\right)\right| \frac{1}{\pi(2 h+2)(2 h+1)} \\
& \quad<\frac{\omega\left(\pi / k ; f^{(r)}\right)}{(2 h+2)(2 h+1)} \leqslant \frac{\omega\left(\pi / n ; f^{(r)}\right)}{(2 h+2)(2 h+1) \pi} .
\end{aligned}
$$

Thus, the replacement gives an error in the sum of these terms which is less than $c \omega\left(\pi / n ; f^{(r)}\right)$.

For simplicity, we suppose $[k / n]-1$ is even. It suffices, then, to consider 


$$
\sum_{h=([k / n]-1) / 2}^{[(k-2) / 2]} \frac{f^{(r)}\left(x+\frac{\theta+2 h \pi}{k}\right)-f^{(r)}\left(x+\frac{\theta+(2 h+1) \pi}{k}\right)}{(2 h+2) \pi}, \quad \pi<\theta<2 \pi .
$$

Let

$$
\Delta_{k}^{h}=\left|f^{(r)}\left(x+\frac{2 h \pi+\theta}{k}\right)-f^{(r)}\left(x+\frac{\theta+(2 h+1) \pi}{k}\right)\right|
$$

and let $\varepsilon_{j}$ be a positive sequence decreasing to zero as slowly as necessary to have $\sum_{k=1}^{\infty} \Phi^{*}\left(1 / k \varepsilon_{k}\right)<\infty$.

For proper choice of $b>0$,

$$
\frac{1}{2 \pi} \sum_{h} \frac{b \Delta_{k}^{h}}{(h+1) \varepsilon_{h+1}}<\sum_{h} \Phi\left(b \Delta_{k}^{h}\right)+\sum_{h} \Phi^{*}\left(\frac{1}{(h+1) \varepsilon_{h+1}}\right)<A<\infty .
$$

Choose $m=m(k, n)<(k-2) / 2$. Then

$$
\frac{1}{2 \pi} \sum_{h=m}^{[(k-2) / 2]} \frac{b \Delta_{k}^{h}}{h+1}<A \varepsilon_{m+1}
$$

and

$$
\begin{aligned}
\frac{1}{2 \pi} \sum_{h=1}^{[(k-2) / 2]} \frac{\Delta_{k}^{h}}{h+1} & <\omega\left(\pi / k ; f^{(r)}\right) \sum_{h=1}^{m-1} \frac{1}{h+1}+\frac{A \varepsilon_{m+1}}{b} \\
& <\omega\left(\pi / k ; f^{(r)}\right) \log m+\frac{A \varepsilon_{m+1}}{b} .
\end{aligned}
$$

We choose $m$ increasing with $n$ so that

$$
\omega\left(\pi / n ; f^{(r)}\right) \log m \rightarrow 0
$$

Thus

$$
\sum \frac{\varphi_{x}\left(\frac{\theta+2 h \pi}{k}\right)-\varphi_{x}\left(\frac{\theta+(2 h+1) \pi}{k}\right)}{\theta+2 h \pi}=o(1) .
$$

Similarly we may show that

$$
J_{n,-}^{(k)}(x)=\int_{-\pi}^{-\pi / n} \frac{\sin k t}{t} \varphi_{x}(t) d t=o(1)
$$

uniformly in $x$ as $n \rightarrow \infty$, and hence, $J_{n}^{(k)}(x)=o(1)$ as $n \rightarrow \infty$ uniformly in $x$.

Now, if we use an argument similar to that in Bari [1, Vol. I, \$32] 


$$
\begin{aligned}
H_{n}^{(k)}(x) & =-\int_{\pi / n<|t|<\pi} \frac{\cos \left(k+\frac{1}{2}\right) t}{2 \sin \frac{1}{2} t} \varphi_{x}(-t) d t \\
& =\int_{\pi / n<|t|<\pi} \frac{\cos \left(k+\frac{1}{2}\right) t}{2 \sin \frac{1}{2} t} \varphi_{x}(t) d t \\
& =\int_{\pi / n<|t|<\pi} \frac{\cos k t}{t} \varphi_{x}(t) d t+o(1)
\end{aligned}
$$

uniformly in $x$ as $n \rightarrow \infty$. Letting

$$
H_{n,+}^{(k)}(x)=\int_{\pi / n}^{\pi} \frac{\cos k t}{t} \varphi_{x}(t) d t
$$

we may use an argument similar to that used for $J_{n,+}^{(k)}(x)$ to show $H_{n,+}^{(k)}(x)=$ $o(1)$ and $H_{n,-}^{(k)}(x)=o(1)$ uniformly in $x$. Therefore,

$$
\left|f(x)-S_{n}(f ; x)\right|=\frac{1}{\pi} \frac{1}{(n+1)^{r}} \cdot o(1)+o(1) \sum_{k=n+1}^{\infty}\left(k^{-r}-(k+1)^{-r}\right)
$$

yielding $\left\|f-S_{n}(f)\right\|_{c}=o\left(n^{-r}\right)$.

1.2 THEOREM. If $f^{(r)} \in \mathfrak{T}_{\Phi}^{*}, 0<r<\infty, \Sigma \Phi^{*}(1 / k)<\infty$, then

$$
\left\|f-S_{n}(f)\right\|_{C}=o\left(n^{-r}\right)+O\left(n^{-r}\left\|f^{(r)}\right\|_{\Phi_{v}}^{*}\right)
$$

where

$$
\|g\|_{\Phi_{0}}^{*}=\lim _{\delta \rightarrow 0+} \sup \left\{\sum\left(g\left(x_{i}\right)-g\left(x_{i-1}\right)\right) b_{i}:|Q|<\delta, \sum \Phi^{*}\left(b_{i}\right)<1\right\} .
$$

PRoof. For $g \in \mathcal{V}_{\Phi}^{*}$ we define

$$
v(\delta, g)=\sup \left\{\sum\left(g\left(x_{i}\right)-g\left(x_{i-1}\right)\right) b_{i}:|Q|<\delta, \sum \Phi^{*}\left(b_{i}\right)<1\right\}
$$

where the $Q$ are partitions of a period. Then

$$
\|g\|_{\Phi_{0}}^{*}=\lim _{\delta \rightarrow 0+} v(\delta, g) \text { and } \omega(\delta, g)<c v(\delta, g)
$$

where $c=1$ if $\Phi^{*}(1)<1, c=1 / \Phi^{*}(1)$ if $\Phi^{*}(1)>1$.

Since the proof is similar to that of Theorem 1.1, we shall simply state where it differs.

Equation (2) becomes

$$
\frac{1}{\pi} \int_{0}^{\pi / n} D_{n}^{(r)}(t) \varphi_{x}(-t) d t=O\left(v\left(\pi / n, f^{(r)}\right) n^{-r}\right) .
$$

We now want to show

$$
\begin{aligned}
\int_{\pi / n<|t|<\pi} A_{k}^{(r)}(t) \varphi_{x}(-t) d t & =O\left(v\left(\pi / n ; f^{(r)}\right)\right)+o(1) \\
& =O\left(\left\|f^{(r)}\right\|_{\Phi_{v}}^{*}\right)+o(1) .
\end{aligned}
$$


Equations (6) and (7) remain the same. For (*) however, we do not use the mean value theorem, and will assume from here that the sums we work with will be integrated over $(\pi, 2 \pi)$. We say again assume that the number of terms in the summation is even, since

$$
\left|\int \sin u \varphi_{x}\left(\frac{u+k \pi}{k}\right) \frac{1}{u+k \pi}\right|<C \frac{1}{k}=o(1) \text { as } n \rightarrow \infty .
$$

Then equation (8) becomes

$$
\text { (8)' }\left|\varphi_{x}\left(\frac{u+(2 h+1) \pi}{k}\right)\left(\frac{1}{u+2 h \pi}-\frac{1}{u+(2 h+1) \pi}\right)\right|<\frac{v\left(\frac{(2 h+3) \pi}{k} ; f^{(r)}\right)}{(2 h+1)(2 h+2) \pi} \text {, }
$$

choosing $M$ as before, the sum of the first $M$ of these terms is

$$
<c v\left(c M / k+o(1) ; f^{(r)}\right)=c v\left(o(1) ; f^{(r)}\right)
$$

as $n \rightarrow \infty$. The sum of the remaining terms is

$$
<\frac{c \sup \left|f^{(r)}\right|}{M+[k / n]}=o(1)
$$

as $n \rightarrow \infty$. If we consider

$$
\left|\frac{\varphi_{x}\left(\frac{u+2 h \pi}{k}\right)-\varphi_{x}\left(\frac{u+(2 h+1) \pi}{k}\right)}{u+2 h \pi}\right|
$$

and replace $u+2 h \pi$ in the denominator with $(2 h+2) \pi$, there is an error

$$
<\frac{v\left(\pi / k ; f^{(r)}\right)}{(2 h+1)(2 h+2) \pi} \leqslant \frac{v\left(\pi / n ; f^{(r)}\right)}{(2 h+1)(2 h+2) \pi} .
$$

Thus, replacement gives an error in the sum of these terms which is less than $\operatorname{cv}\left(\pi / n ; f^{(r)}\right)$.

Equations (11) and (12) remain the same, but (13) becomes, with the use of Hölder's inequality

$$
\begin{aligned}
& \frac{1}{2 \pi} \sum_{h=([k / n]-1) / 2}^{[(k-2) / 2]} \frac{\Delta_{k}^{h}}{h+1}<\frac{1}{2 \pi} \sum_{h=0}^{[(k-2) / 2]} \frac{\Delta_{k}^{h}}{h+1} \\
& \quad<v\left(\frac{\pi}{k} ; f^{(r)}\right)\left\|\left\{\frac{1}{h+1}\right\}_{h=0}^{[(k-2) / 2]}\right\|_{\Phi^{*}}^{0}<c v\left(\pi / k ; f^{(r)}\right),
\end{aligned}
$$

where

$$
\left\|\left\{a_{j}\right\}_{j-1}^{p}\right\|_{\Phi}^{0}=\sup \left\{\sum_{i=1}^{p} a_{i} b_{i}: \sum_{i=1}^{p} \Phi^{*}\left(b_{i}\right) \leqslant 1\right\} .
$$

A short digression seems to be in order to justify this last step. 
Consider $\mathbf{R}^{p}$ and let $a=\left(a_{1}, \ldots, a_{p}\right), b=\left(b_{1}, \ldots, b_{p}\right) \in \mathbf{R}^{p}$, and define

$$
\|a\|_{\Phi}^{0}=\sup \left\{\sum_{i=1}^{p} a_{i} b_{i}: \sum \Phi^{*}\left(b_{i}\right) \leqslant 1\right\} .
$$

For any $a, b \in \mathbf{R}^{p}$,

$$
\sum_{i=1}^{p} a_{i} b_{i} \leqslant\|a\|_{\Phi}^{0}\|b\|_{\Phi *}^{0}
$$

Thus, let $p=[(k-2) / 2]+1$.

Let

$$
a=\left\{a_{h+1}=\Delta_{k}^{h}\right\}_{h=0}^{p-1}, \quad b^{p}=\left\{b_{i}=1 /(i+1)\right\}_{i=0}^{p-1}
$$

Then

$$
\sum_{i=0}^{[(k-2) / 2]} \frac{\Delta_{k}^{i}}{(i+1)} \leqslant\|a\|_{\Phi}^{0}\left\|b^{p}\right\|_{\Phi^{*}}^{0}
$$

Now, since $\sum_{i=0}^{\infty} \Phi^{*}(1 /(i+1))<\infty$,

$$
\lim _{p \rightarrow \infty}\left\|b^{p}\right\|_{\Phi^{*}}^{0}=A<\infty
$$

and

$$
\begin{aligned}
\|a\|_{\Phi}^{0} & =\sup \left\{\sum _ { h = 0 } ^ { p } b _ { i } \left(f^{(r)}\left(x+\frac{\theta}{k}+\frac{2 h \pi}{k}\right)\right.\right. \\
& \left.\left.\quad-f^{(r)}\left(x+\frac{\theta}{k}+\frac{(2 h+1) \pi}{k}\right)\right): \sum \Phi^{*}\left(b_{i}\right)<1\right\} \\
& <v\left(\pi / k ; f^{(r)}\right) .
\end{aligned}
$$

Putting these two results together yields

$$
\sum_{i=0}^{[(k-2) / 2]} \frac{\Delta_{k}^{i}}{(i+1)} \leqslant A v\left(\pi / k ; f^{(r)}\right) .
$$

Thus,

$$
\begin{aligned}
\left|L_{n}^{(k)}(x)\right| & <\int_{\pi}^{2 \pi} \sin u\left(\operatorname{cv}\left(\pi / n, f^{(r)}\right)+o(1)\right) d u \\
& <\operatorname{cv}\left(\pi / n ; f^{(r)}\right)+o(1),
\end{aligned}
$$

and we can show that

$$
\int_{\pi / n<|t|<\pi} A_{k}^{(r)}(t) \varphi_{x}(-t) d t=O\left(\left\|f^{(r)}\right\|_{\Phi_{v}}^{*}\right)+o(1)
$$


and the conclusion follows from equation (3).

2. Approximation in the Orlicz space norm. The theorem which we shall prove in this section is a generalization of the fundamental result of $S$. M. Nikol'skii [8] and B. I. Golubov [3].

THEOREM. Let $f \in \mathcal{V}_{\Phi}^{(r)}, \Phi$ an $N$-function, $r \geqslant 0$. Further suppose $\left\{x_{i}\right\}$ are the points of $[-\pi, \pi)$ at which $f^{(r)}$ is discontinuous. Let

$$
\sigma_{i}=f^{(r)}(x+0)-f^{(r)}(x-0) .
$$

Then, for each reflexive $\Delta^{\prime} N$-function, $\Psi$, with $\int_{1}^{\infty} \Psi\left(t^{-1}\right) d t<\infty$ and

$$
\lim _{x \rightarrow 0} \Psi(x) / \Phi(x)=0
$$

we have, for $f^{(r)}$ continuous,

$$
\left\|f-S_{n}(f)\right\|_{\Psi}=o\left(1 / n^{r} \Psi^{-1}(n)\right)
$$

otherwise,

$$
\left\|f-S_{n}(f)\right\|_{\Psi} \leqslant \frac{1}{n^{r} \Psi^{-1}(n)}\left[o(1)+1 / \Psi^{-1}\left(1 / \frac{C_{\Psi}^{2} S}{2} \sum_{j=1}^{\infty} \Psi\left(\sigma_{j}\right)\right)\right] .
$$

To prove Theorem 2.1 we must first develop several other results.

Let $\alpha$ be an arbitrary real number, $n$ any nonnegative integer, and $r$ any nonnegative real number. Then we define

$$
\varphi_{n}(\alpha, r, x)=\sum_{k=n+1}^{\infty} \frac{\cos (k x+\alpha)}{k^{r+1}} .
$$

2.1 LeMMA. For $\Phi$ a $\Delta^{\prime} N$-function such that $\int_{|t|>1} \Phi\left(t^{-1}\right)<\infty$, and for $r>0$,

$$
\varphi_{n}(\alpha, r, x)=\frac{1}{\Gamma(r+1) n^{r}} \varphi_{*}(\alpha, r, n x)+\varepsilon_{n}(\alpha, r, x)
$$

where

$$
\varphi_{*}(\alpha, r, t)=\int_{0}^{\infty} e^{-v} v^{r} \frac{(v \cos (t+\alpha)-t \sin (t+\alpha))}{v^{2}+t^{2}} d v
$$

and

$$
\int_{-\pi}^{\pi} \Phi\left(n^{r} \Phi^{-1}(n) \varepsilon_{n}(\alpha, r, x)\right) d x=O\left(\Phi\left(\Phi^{-1}(n) / n\right)\right) .
$$

To prove Lemma 2.1 we first need several auxiliary results.

2.1A LeMMA. For $v<n,|t|<n \pi$, 
(3)

$$
\begin{aligned}
& \frac{1}{\frac{t^{2}}{n^{2}} \exp \left(\frac{-v}{n+1}\right)+\left(1-\exp \left(\frac{-v}{n+1}\right)\right)^{2}}-\frac{1}{\frac{v^{2}+t^{2}}{n^{2}}} \mid \\
& =O\left(\frac{1+v}{n}\right) / \frac{v^{2}+t^{2}}{n^{2}}
\end{aligned}
$$

Proof. The left-hand side of (3) equals

$$
\left|\frac{\frac{v^{2}}{n^{2}}+\frac{t^{2}}{n^{2}}\left(1-\exp \left(\frac{-v}{n+1}\right)\right)-\left(1-\exp \left(\frac{-v}{n+1}\right)\right)^{2}}{\left(\left(1-\exp \left(\frac{-v}{n+1}\right)\right)^{2}+\frac{t^{2}}{n^{2}} \exp \left(\frac{-v}{n+1}\right)\right)\left(\frac{v^{2}+t^{2}}{n^{2}}\right)}\right|
$$

If we use the substitution

$$
1-\exp \left(\frac{-v}{n+1}\right)=\frac{v}{n+1}+O\left(\frac{v^{2}}{n^{2}}\right)=\frac{v}{n}+O\left(\frac{v+v^{2}}{n^{2}}\right)
$$

in the above equation, the numerator becomes

$$
\begin{aligned}
& \left|\frac{v^{2}}{n^{2}}+\frac{t^{2}}{n^{2}}\left(1-\exp \left(\frac{-v}{n+1}\right)\right)-\left(1-\exp \left(\frac{-v}{n+1}\right)\right)^{2}\right| \\
& =\mid \frac{v^{2}}{n^{2}}+\frac{t^{2}}{n^{2}}\left(\frac{v}{n}+O\left(\frac{v+v^{2}}{n^{2}}\right)\right)-\frac{v^{2}}{n^{2}} \\
& +O\left(\frac{v^{2}+v^{3}}{n^{3}}\right)+O\left(\frac{v^{2}+2 v^{3}+v^{4}}{n^{4}}\right) \\
& =O\left(\frac{v^{2}}{n^{3}}\right)+O\left(\frac{v t^{2}}{n^{3}}\right)+O\left(\frac{v^{3}}{n^{3}}\right)=O\left(\frac{v^{2}+t^{2} v+v^{3}}{n^{3}}\right) \text {. }
\end{aligned}
$$

On the other hand,

$$
1-\exp \left(\frac{-v}{n+1}\right)>\frac{v}{n+1}>\frac{v}{2 n}
$$

and

$$
\frac{t^{2}}{n^{2}} \exp \left(\frac{-v}{n+1}\right)>\frac{t^{2}}{n^{2}} \exp (-1)
$$

Using the above approximations, the left side of (3) is

$$
\begin{aligned}
& <\frac{o\left(\frac{v^{2}+t^{2} v+v^{3}}{n^{3}}\right)}{\left(\frac{v^{2}}{4 n^{2}}+e^{-1} \frac{t^{2}}{n^{2}}\right)\left(\frac{v^{2}+t^{2}}{n^{2}}\right)} \\
& <\frac{O\left(\left(v^{2}+t^{2} v+v^{3}\right) / n\right)}{\left(v^{2}+t^{2}\right)^{2} / n^{2}}<\frac{o((1+v) / n)}{\left(v^{2}+t^{2}\right) / n^{2}} .
\end{aligned}
$$


2.1B LEMMA. If $0<\rho<1,|x|<\pi$, then

(4)

$$
\left|\frac{1}{(1-\rho)^{2}+4 \rho \sin ^{2} x / 2}-\frac{1}{(1-\rho)^{2}+\rho x^{2}}\right|=\frac{O\left(x^{2}\right)}{(1-\rho)^{2}+\rho x^{2}} .
$$

Proof. The left side of (4) equals

$$
\left|\frac{\rho x^{2}\left(1+\frac{2 \sin x / 2}{x}\right)\left(1-\frac{2 \sin x / 2}{x}\right)}{\left((1-\rho)^{2}+4 \rho \sin ^{2} x / 2\right)(1-\rho)^{2}+\rho x^{2}}\right| .
$$

Now, for $x \neq 0$,

$$
\left(1+\frac{2}{x} \sin \frac{x}{2}\right)<c \text { and }\left(1-\frac{2}{x} \sin \frac{x}{2}\right)=O\left(x^{2}\right) .
$$

Hence, the left side of (4) equals

$$
\left|\frac{\rho O\left(x^{4}\right)}{\left((1-\rho)^{2}+4 \rho \sin ^{2} x / 2\right)\left((1-\rho)^{2}+\rho x^{2}\right)}\right|<\frac{O\left(x^{2}\right)}{(1-\rho)^{2}+\rho x^{2}} .
$$

2.1C LemMa. When $0<\rho<1$ and $|x|<\pi$,

$$
\begin{aligned}
\cos ((n+1) x+\alpha)-\rho \cos (n x+\alpha) \\
=(1-\rho) \cos (n x+\alpha)-x \sin (n x+\alpha)+O\left(x^{2}\right) .
\end{aligned}
$$

Proof.

$$
\begin{aligned}
& \cos ((n+1) x+\alpha)-\rho \cos (n x+\alpha) \\
&= \cos x \cos (n x+\alpha)-\rho \cos (n x+\alpha)-\sin x \sin (n x+\alpha) \\
&= \cos (n x+\alpha)+O\left(x^{2}\right) \cos (n x+\alpha)-\rho \cos (n x+\alpha) \\
&-x \sin (n x+\alpha)+O\left(x^{3}\right) \sin (n x+\alpha) \\
&=(1-\rho) \cos (n x+\alpha)-x \sin (n x+\alpha)+O\left(x^{2}\right) .
\end{aligned}
$$

2.1D LemMa. Let $\Phi$ be a $\Delta_{2} N$-function such that $\int_{|t|>1} \Phi\left(t^{-1}\right) d t<\infty$. Define for $r>0$,

$$
h_{r}(t)=\int_{0}^{\infty} \frac{e^{-v} v^{r}}{v^{2}+t^{2}} d v
$$

and

$$
g_{r}(t)=t \int_{0}^{\infty} \frac{e^{-v} v^{r}}{v^{2}+t^{2}} d v=t h_{r}(t) .
$$

Then there exist constants $C=C(\Phi, r)$ and $B=B(\Phi, r)$ such that for $r>1$

$$
\int_{-\infty}^{\infty} \Phi\left(h_{r}(t)\right) d t \leqslant C,
$$

and for $r>0$ 
52

ELAINE COHEN

$$
\int_{-\infty}^{\infty} \Phi\left(g_{r}(t)\right) d t<B
$$

Proof. We have that for $0<t<\infty, r>0$,

$$
h_{r}(t)<\frac{1}{t^{2}} \int_{0}^{\infty} e^{-v} v^{r} d v<\frac{C(r)}{t^{2}}=\frac{C}{t^{2}}
$$

and for $0<t<1$,

$$
\begin{aligned}
h_{r}(t) & =\int_{0}^{\infty} \frac{e^{-u t}(u t)^{r} t d u}{t^{2}\left(1+u^{2}\right)} \\
& <\frac{1}{t^{2}} \int_{0}^{1} e^{-u t}(u t)^{r} t d u+\int_{1}^{\infty} e^{-u t}(u t)^{r-2} t d u \\
& =\frac{1}{t^{2}} \int_{0}^{t} e^{-v} v^{r} d v+\int_{t}^{1} e^{-v} v^{r-2} d v+\int_{1}^{\infty} e^{-v} v^{r-2} d v \\
& <\frac{1}{t^{2}} \int_{0}^{t} v^{r} d v+\int_{t}^{1} v^{r-2} d v+C \\
& < \begin{cases}C\left(t^{r-1}+1\right), & r \neq 1, r>0, \\
C(1+|\log t|), & r=1 .\end{cases}
\end{aligned}
$$

Since $\Phi\left(c / t^{2}\right)=O\left(c / t^{2}\right)=O\left(1 / t^{2}\right)$ as $t \rightarrow \infty$, we have

$$
\int_{|t|>1} \Phi\left(h_{r}(t)\right) d t<\int_{|t|>1} \Phi\left(\frac{c}{t^{2}}\right) d t=\int_{|t|>1} O\left(\frac{1}{t^{2}}\right) d t=I_{1}<\infty .
$$

If $r>1$, we have

$$
\int_{|t|<1} \Phi\left(h_{r}(t)\right) d t<\int_{|t|<1} \Phi\left(c\left(1+|t|^{r-1}\right)\right) d t<\mathrm{I}_{2}<\infty
$$

If $r=1$, we have

$$
\int_{|t|<1} \Phi\left(h_{r}(t)\right) d t \leqslant \int_{|t|<1} \Phi(c+c|\log | t||) d t \leqslant c+\frac{1}{2} \int_{|t|<1} \Phi(2 c \log |t|) d t .
$$

Now since $\Phi$ is $\Delta_{2}$ for large values we know that there exists $u_{0}$ and $\beta>1$ such that $u>u_{0}$ implies $\Phi(u)<A\left(u_{0}, \Phi\right) u^{\beta}$. Let $\alpha=\beta+1$. Then $|\log | t||=$ $o\left(t^{-1 / \alpha}\right)$ as $t \rightarrow 0$. Choose $2>\varepsilon>0$. Then there exists $\delta=\delta\left(u_{0}, \varepsilon\right), \delta<$ $u_{0}^{-\alpha}$, such that for $|t|<\delta$,

$$
2 c t^{1 / \alpha}|\log t|<\varepsilon / 2
$$

and 


$$
\begin{aligned}
\int_{|t|<1} \Phi(2 c \log |t|) d t & <\int_{\delta<|t|<1} \Phi(2 c \log |t|) d t+\frac{\varepsilon}{2} \int_{|t|<\delta} \Phi\left(t^{-1 / \alpha}\right) d t \\
& <A_{1}+\frac{\varepsilon}{2} \int_{u>\delta^{-1 / \alpha}} \alpha \Phi(u) / u^{(1+\alpha)} d u \\
& =A_{1}+\frac{\varepsilon}{2} \int_{|u|>\delta^{-1 / \alpha}} \alpha \Phi(u) u^{-\beta-2} d u \\
& <A_{1}+\frac{\varepsilon \alpha A}{2} \int_{|u|>\delta^{-1 / \alpha}} u^{-2} d u=I_{2}<\infty .
\end{aligned}
$$

Thus, we have that

$$
\int_{-\infty}^{\infty} \Phi\left(h_{r}(t)\right) d t<\infty \text { for } r>1
$$

Now, suppose $r>0$, then for $r \neq 1$,

$$
\int_{0<|t|<1} \Phi\left(g_{r}(t)\right) d t<\int_{0<|t|<1} \Phi\left(c\left(t+t^{r}\right)\right) d t<\infty,
$$

and for $r=1$,

$$
\int_{0<|t|<1} \Phi\left(g_{r}(t)\right) d t<\frac{1}{2} \int_{0<|t|<1} \Phi(2 c t) d t+\frac{1}{2} \int_{0<|t|<1} \Phi(2 c t \log |t|) d t
$$

since

$$
\lim _{t \rightarrow 0}|t \log | t||=0,
$$

$|t||\log | t||$ is bounded on $(-1,1)$. Hence, $\Phi(2 c t \log |t|)$ is bounded on $(-1,1)$ and has a finite integral. Thus,

$$
\int_{0<|t|<1} \Phi\left(g_{r}(t)\right) d t<\infty, \quad r>0 .
$$

For all $r \geqslant 0$, we have, by hypothesis,

$$
\int_{|t|>1} \Phi\left(g_{r}(t)\right) d t<\int_{|t|>1} \Phi(c / t) d t<\infty .
$$

Thus, for $r \geqslant 0$,

$$
\int_{-\infty}^{\infty} \Phi\left(g_{r}(t)\right) d t<\infty
$$

We now return to the proof of Lemma 2.1. From Nikol'skin [8] we have that

$$
\begin{aligned}
& \varphi_{n}(\alpha, r, x) \\
& \quad=\frac{1}{\Gamma(r+1)} \int_{0}^{1} \rho^{n}\left(\log \frac{1}{\rho}\right)^{r} \frac{\cos ((n+1) x+\alpha)-\rho \cos (n x+\alpha)}{\rho^{2}-2 \beta \cos x+1} d \rho .
\end{aligned}
$$

By Lemmas 2.1B and 2.1C, 


$$
\begin{aligned}
& \frac{\cos ((n+1) x+\alpha)-\rho \cos (n x+\alpha)}{\rho^{2}-2 \rho \cos x+1} \\
& \quad=\frac{\cos (n x+\alpha)(1-\rho)-x \sin (n x+\alpha)}{(1-\rho)^{2}+\rho x^{2}}+\frac{O\left(x^{2}\right)}{(1-\rho)^{2}+\rho x^{2}} .
\end{aligned}
$$

We may write

$$
\begin{aligned}
& \varphi_{n}(\alpha, r, x) \\
& =\frac{1}{\Gamma(r+1)} \int_{0}^{1} \rho^{n}\left(\log \frac{1}{\rho}\right)^{r} \frac{\cos (n x+\alpha)(1-\rho)-x \sin (n x+\alpha)}{(1-\rho)^{2}+\rho x^{2}} d \rho \\
& \quad+\delta_{n}(r, x) \\
& \equiv \theta_{n}(\alpha, r, x)+\delta_{n}(r, x),
\end{aligned}
$$

and

$$
\begin{aligned}
\left|\delta_{n}(r, x)\right| & =\left|O\left(x^{2}\right) \int_{0}^{1} \frac{\rho^{n}(\log 1 / \rho)^{r}}{(1-\rho)^{2}+\rho x^{2}} d \rho\right| \\
& <\left|O(1) \int_{0}^{1} \rho^{n-1}(\log 1 / \rho)^{r} d \rho\right| \\
& =O\left(n^{-r-1}\right) .
\end{aligned}
$$

Now consider $\theta_{n}(\alpha, r, x)$. We substitute $\rho=\exp (-v /(n+1))$ and $t=n x$ in the expression defining $\theta_{n}$, obtaining

$$
\theta_{n}\left(\alpha, r, \frac{t}{n}\right)=\frac{1}{\Gamma(r+1) n^{r+1}} \int_{0}^{\infty} e^{-v} v_{r} \frac{\left(1-e^{-v /(n+1)}\right) \cos (t+\alpha)-t / n \sin (t+\alpha)}{\left(1-e^{-v /(n+1))^{2}}+\frac{t^{2}}{n^{2}} e^{-v /(n+1)}\right.} d v .
$$

Now let

$$
\theta_{n}(\alpha, r, t / n)=\frac{1}{\Gamma(r+1)(n+1)^{r+1}} \int_{0}^{0} \cdots d v+\tilde{\delta}_{n}(\alpha, r, t)
$$

where

$$
\begin{aligned}
\left|\tilde{\delta}_{n}(\alpha, r, t)\right| & <\frac{1}{(n+1)^{r+1} \Gamma(r+1)} \cdot \frac{\pi+1}{\left(1-e^{-n /(n+1)}\right)^{2}} \int_{n}^{\infty} e^{-v} v^{r} d v \\
& <c \exp (-n / 2)
\end{aligned}
$$

where $c$ depends only on $r$.

Using Lemma 2.1A and

$$
1-\exp \left(\frac{-v}{n+1}\right)=\frac{v}{n}+O\left(\frac{v+v^{2}}{n^{2}}\right)
$$


DEGREE OF APPROXIMATION OF A FUNCTION

55

we have

$$
\begin{aligned}
& \theta_{n}(\alpha, r, t / n) \\
& =\tilde{\delta}_{n}(\alpha, r, t)+\frac{1}{\Gamma(r+1)(n+1)^{r+1}} \\
& \cdot \int_{0}^{n} \frac{e^{-v} v^{r}}{n}\left(\frac{v \cos (t+\alpha)-t \sin (t+\alpha)+o\left(\frac{v+v^{2}}{n}\right)-\left(1+o\left(\frac{1+v}{n}\right)\right)}{\frac{v^{2}+t^{2}}{n^{2}}}\right) d x \\
& =\tilde{\delta}_{n}(\alpha, r, t)+\frac{1}{\Gamma(r+1)(n+1)^{r+1}} \int_{0}^{n} e^{-v} \frac{v^{r} O((1+v)|t|)}{v^{2}+t^{2}} d v \\
& +\frac{1}{\Gamma(r+1)(n+1)^{r+1}} \int_{0}^{n} e^{-v} \frac{v^{r} O\left(v+v^{2}\right)}{v^{2}+t^{2}} d v \\
& -\frac{1}{\Gamma(r+1) n^{r}} \int_{n}^{\infty} e^{-v} \frac{v^{r}(v \cos (t+\alpha)-t \sin (t+\alpha))}{v^{2}+t^{2}} d v \\
& +\left(\frac{n}{(n+1)^{r+1}}-\frac{1}{n^{r}}\right) \frac{1}{\Gamma(r+1)} \int_{0}^{n} e^{-v} \frac{v^{r}(v \cos (t+\alpha)-t \sin (t+\alpha))}{v^{2}+t^{2}} d v \\
& +\frac{1}{n^{r}} \frac{1}{\Gamma(r+1)} \int_{0}^{\infty} e^{-v} \frac{v^{r}(v \cos (t+\alpha)-t \sin (t+\alpha))}{v^{2}+t^{2}} d v \\
& =\tilde{\delta}_{n}(\alpha, r, t)+\mathrm{I}_{r}(t)+\mathrm{II}_{r}(t)-\mathrm{III}_{r}(t)+\mathrm{IV}_{r}(t)+\varphi_{*}(\alpha, r, t) \\
& =\varepsilon_{n}^{\prime}(\alpha, r, t)+\varphi_{*}(\alpha, r, t) \text {. }
\end{aligned}
$$

Using Lemma 2.1D, we have that,

$$
\begin{aligned}
\left|\mathrm{IV}_{r}(t)\right| \leqslant & \left|\frac{n}{(n+1)^{r+1}}-\frac{1}{n^{r}}\right|\left|\frac{1}{\Gamma(r+1)}\right| \\
& \cdot\left|h_{r+1}(t) \cos (t+\alpha)-g_{r}(t) \sin (t+\alpha)\right|
\end{aligned}
$$

so

$$
\begin{aligned}
\int_{-n \pi}^{n \pi} \Phi\left(n^{r} \Phi^{-1}(n) \mathrm{IV}_{r}(t)\right) & <c_{\Phi, r} \Phi\left(\Phi^{-1}(n) / n\right) \int_{0}^{n \pi} \Phi\left(\left|h_{r+1}(t)\right|+\left|g_{r}(t)\right|\right) d t \\
& <c \Phi\left(\Phi^{-1}(n) / n\right)=O\left(\Phi\left(\Phi^{-1}(n) / n\right)\right) .
\end{aligned}
$$

We note that

$$
O\left(v+v^{2}\right)= \begin{cases}O\left(v^{2}\right), & v>1 \\ O(v), & v<1\end{cases}
$$

We first consider 


$$
\left|\mathrm{III}_{r}(t)\right| \leqslant \frac{1}{n^{r} \Gamma(r+1)} \int_{n}^{\infty} \frac{e^{-v} v^{r}(2 v)}{v^{2}} d v=O\left(e^{-n / 2}\right) .
$$

Now, suppose $r>0$. Then

$$
\begin{aligned}
\left|(n+1)^{r+1} \Gamma(r+1) \mathrm{I}_{r}(t)\right| & =\int_{0}^{n} \frac{e^{-v} v^{r} O((1+v)|t|)}{v^{2}+t^{2}} d v \\
& \left.=\iint_{0<|t|<v<n}+\int_{\substack{0<v<|t| \\
v<n}}\right] e^{-v} \frac{v^{r} O((1+v)|t|)}{v^{2}+t^{2}} d v .
\end{aligned}
$$

Let the quantity represented by the first integral on the right be $A$ and the second be $B$. Then,

$$
\begin{aligned}
A & \leqslant \int_{0<|t|<v<n} e^{-v} \frac{\left(O\left(v^{r+1}\right)+O\left(v^{r+2}\right)\right)}{v^{2}+t^{2}} d v \\
& <\int_{0}^{n} O\left(e^{-v} v^{r-1}\right) d v+\int_{0}^{n} O\left(e^{-v} v^{r}\right) d v \leqslant C . \\
B & \leqslant \int_{0}^{|t|} e^{-v} \frac{v^{r} O((1+v)|t|)}{v^{2}+t^{2}} d v \leqslant \int_{0}^{|t|} e^{-v} \frac{v^{r} O(1+v)}{|t|} d v \\
& \leqslant \int_{0}^{|t|} e^{-v} \frac{v^{r} O(1+v)}{v} d v=\int_{0}^{|t|} e^{-v} v^{r-1} O(1+v) d v \leqslant C .
\end{aligned}
$$

The constants, $C$, in $A$ and $B$ depend only on $r$. Hence

$$
\left|I_{r}(t)\right|=O\left((n+1)^{-r-1}\right) .
$$

Also,

$$
\begin{aligned}
\left|(n+1)^{r+1} \Gamma(r+1) \mathrm{II}_{r}(t)\right| & =\int_{0}^{1} \frac{e^{-v} v^{r} O(v)}{v^{2}+t^{2}} d v+\int_{1}^{n} \frac{e^{-v} v^{r} O\left(v^{2}\right)}{v^{2}+t^{2}} d v \\
& \leqslant C+\int_{1}^{n} e^{-v} O\left(v^{2}\right) d v=O(1) .
\end{aligned}
$$

Thus, $\left|\mathrm{II}_{r}(t)\right|=O\left((n+1)^{-r-1}\right)$. Hence,

$$
\begin{aligned}
\int_{-n \pi}^{n \pi} \Phi & \left(n^{r} \Phi^{-1}(n) \varepsilon_{n}^{\prime}(\alpha, r, t)\right) d t \\
\leqslant & \frac{1}{2} \int_{-n \pi}^{n \pi} \Phi\left(2 n^{r} \Phi^{-1}(n)\left(\tilde{\delta}+\mathrm{I}_{r}+\mathrm{II}_{r}-\mathrm{III}_{r}\right)\right) d t \\
& +\frac{1}{2} \int_{-n \pi}^{n \pi} \Phi\left(2 n^{r} \Phi^{-1}(n) \mathrm{IV}_{r}(t)\right) d t \\
= & O\left(n \Phi\left(\Phi^{-1}(n) / n\right)\right)+O\left(\Phi\left(\Phi^{-1}(n) / n\right)\right) .
\end{aligned}
$$


We now suppose $r=0$. Then

$$
\begin{aligned}
\left|(n+1) \mathrm{II}_{0}(t)\right| & =\int_{0}^{1} e^{-v} \frac{O\left(v+v^{2}\right)}{v^{2}+t^{2}} d v+\int_{1}^{n} e^{-v} \frac{O\left(v+v^{2}\right)}{v^{2}+t^{2}} d v \\
& \leqslant \int_{0}^{1} \frac{e^{-v} O\left(v+v^{2}\right)}{v^{2}+t^{2}} d v+O(1) .
\end{aligned}
$$

For $|t| \geqslant 1$,

$$
\int_{0}^{1} e^{-v} \frac{O\left(v+v^{2}\right)}{v^{2}+t^{2}} d v \leqslant \int_{0}^{1} \frac{e^{-v} O(v) d v}{t^{2}} \leqslant \int_{0}^{1} e^{-v} O(v) d v=O(1),
$$

and for $0<|t|<1$,

$$
\begin{aligned}
\int_{0}^{1} e^{-v} \frac{O\left(v+v^{2}\right)}{v^{2}+t^{2}} d v & \leqslant \int_{0}^{1} \frac{O(v)}{v^{2}+t^{2}} d v \\
& =O\left(\log \left(\frac{1+t^{2}}{t^{2}}\right)\right)=O\left(\log 2+\log \frac{1}{t}\right) .
\end{aligned}
$$

Thus,

$$
\left|(n+1) I_{0}(t)\right|=O(1)+ \begin{cases}0, & |t|>1 \\ O(\log 1 / t), & |t|<1\end{cases}
$$

Now,

$$
\left|(n+1) \mathrm{I}_{0}(t)\right|=\int_{0}^{1} \frac{e^{-v} O((1+v)|t|)}{v^{2}+t^{2}} d v+\int_{1}^{n} e^{-v} \frac{O((1+v)|t|)}{v^{2}+t^{2}} d v,
$$

where

$$
\int_{1}^{n} \frac{e^{-v} O((1+v)|t|)}{v^{2}+t^{2}} d v \leqslant \int_{1}^{n} \frac{e^{-v} O\left(v^{2}\right)}{v^{2}}+\int_{1}^{n} \frac{e^{-v} O\left(t^{2}\right)}{t^{2}} d v=O(1),
$$

and, if $|t| \geqslant 1$,

$$
\int_{0}^{1} e^{-v} \frac{O((1+v)|t|)}{v^{2}+t^{2}} d v \leqslant \int_{0}^{|t|} \frac{e^{-v} O\left(t^{2}\right)}{t^{2}} d v=O(1)
$$

while for $|t|<1$, 


$$
\begin{aligned}
\int_{0}^{1} e^{-v} \frac{O((1+v)|t|)}{v^{2}+t^{2}} d v< & \int_{0}^{|t|} \frac{e^{-v} O(|t|)}{t^{2}} d v+\int_{|t|}^{1} \frac{e^{-v} O(|t|)}{v^{2}+t^{2}} d v \\
& +\int_{|t|}^{1} \frac{e^{-v} O(v|t|)}{v^{2}+t^{2}} d v \\
& <O(1)+\int_{|t|}^{1} \frac{O(v)}{v^{2}+t^{2}} d v+\int_{|t|}^{1} \frac{e^{-v} O\left(v^{2}\right)}{v^{2}} d v \\
& =O(1)+O\left(\log \left(\frac{1+t^{2}}{2 t^{2}}\right)\right) .
\end{aligned}
$$

Thus,

$$
\left|(n+1) \mathrm{I}_{0}(t)\right|=O(1)+ \begin{cases}0, & |t| \geqslant 1 \\ O(\log |1 / t|), & |t|<1\end{cases}
$$

The above conditions show, if $\varepsilon_{r}^{*}(\alpha, 0, t)=\tilde{\delta}(\alpha, 0, t)+\mathrm{I}_{0}(t)+\mathrm{II}_{0}(t)-$ $\mathrm{III}_{0}(t)$, then

$$
\varepsilon_{r}^{*}(\alpha, 0, t)=O\left(\frac{1}{n+1}\right)+ \begin{cases}0, & |t|>1 \\ O(\log |1 / t| /(n+1)), & |t|<1\end{cases}
$$

and, hence

$$
\begin{aligned}
& \int_{-n \pi}^{n \pi} \Phi\left(\Phi^{-1}(n) \varepsilon_{n}^{*}(\alpha, 0, t)\right) d t \\
& \quad=2 \int_{0}^{1} \Phi\left(\frac{\Phi^{-1}(n)}{n+1} O\left(1+\log \frac{1}{t}\right)\right) d t+\int_{1<|t|<n \pi} \Phi\left(\frac{\Phi^{-1}(n)}{n+1}\right) d t .
\end{aligned}
$$

Substituting $t=e^{-w}$ in the first integral gives

$$
\begin{aligned}
\int_{0}^{1} \Phi\left(\frac{\Phi^{-1}(n)}{n+1}\right. & \left.O\left(1+\log \frac{1}{t}\right)\right) d t=\int_{0}^{\infty} \Phi\left(\frac{\Phi^{-1}(n)}{n+1} \cdot O(1+w)\right) e^{-w} d w \\
& <c \Phi\left(\frac{\Phi^{-1}(n)}{n+1}\right) \int_{0}^{\infty} e^{-w} d w+\int_{0}^{\infty} \Phi\left(\frac{\Phi^{-1}(n)}{n+1} O(w)\right) e^{-w} d w \\
& <c \Phi\left(\frac{\Phi^{-1}(n)}{n+1}\right)+c \Phi\left(\frac{\Phi^{-1}(n)}{n+1}\right) \int_{0}^{\infty} \Phi(w) e^{-w} d w .
\end{aligned}
$$

Now $\Phi$ is $\Delta_{2}$ implies that there exists $u_{0}$ such that for $w>u_{0}, \Phi(w)<A w^{p}$ for some $p>1, A$ a constant. Applying this,

$$
\int_{0}^{\infty} \Phi(w) e^{-w} d w<\int_{0}^{u_{0}} \Phi(w) e^{-w} d w+\int_{u_{0}}^{\infty} A w^{p} e^{-w} d w<\infty .
$$


DEGREE OF APPROXIMATION OF A FUNCTION

59

Hence,

$$
\int_{-n \pi}^{n \pi} \Phi\left(\Phi^{-1}(n) \varepsilon_{n}^{*}(\alpha, 0, t)\right) d t<O\left(n \Phi\left(\Phi^{-1}(n) / n\right)\right) .
$$

Then, since $\varepsilon_{n}^{\prime}(\alpha, 0, t)=\varepsilon_{n}^{*}(\alpha, 0, t)+\operatorname{IV}_{0}(t)$,

$$
\int_{-n \pi}^{n \pi} \Phi\left(\Phi^{-1}(n) \varepsilon_{n}^{\prime}(\alpha, 0, t)\right) d t \leqslant n O\left(\Phi\left(\Phi^{-1}(n) / n\right)\right)+O\left(\Phi\left(\Phi^{-1}(n) / n\right)\right) .
$$

Define $\varepsilon_{n}(\alpha, r, x)=\varepsilon_{n}^{\prime}(\alpha, r, n x)+\delta_{n}(r, x)$, for $|x|<\pi$. Then,

$$
\begin{aligned}
\int_{-\pi}^{\pi} \Phi & \left(n^{r} \Phi^{-1}(n) \varepsilon_{n}(\alpha, n, x)\right) d x \\
& =\int_{-n \pi}^{n \pi} \Phi\left(n^{r} \Phi^{-1}(n)\left(\varepsilon_{n}^{\prime}(\alpha, r, t)+\delta_{n}\left(r, \frac{t}{n}\right)\right)\right) \frac{d t}{n} \\
& =O\left(\Phi\left(\Phi^{-1}(n) / n\right)\right) .
\end{aligned}
$$

Hence,

$$
\begin{aligned}
\varphi_{n}(\alpha, r, x)= & \frac{1}{\Gamma(r+1) n^{r}} \int_{0}^{\infty} e^{-v} v^{r} \frac{(v \cos (n x+\alpha)-(n x) \sin (n x+\alpha))}{v^{2}+(n x)^{2}} d v \\
& +\varepsilon_{n}(\alpha, r, x) \\
= & \varphi_{*}(\alpha, r, n x)+\varepsilon_{n}(\alpha, r, x)
\end{aligned}
$$

where

$$
\int_{-\pi}^{\pi} \Phi\left(n^{r} \Phi^{-1}(n) \varepsilon_{n}(\alpha, r, x)\right) d x=O\left(\Phi\left(\Phi^{-1}(n) / n\right)\right) .
$$

2.2 LemMa. Let $\Phi$ be a $\Delta^{\prime} N$-function such that $\int_{|t|>1} \Phi\left(t^{-1}\right) d t<\infty$, and let $r>0$. Then there exists $S=S(\Phi, \alpha, r)$ such that

$$
\int_{0}^{2 \pi} \Phi\left(n^{r} \Phi^{-1}(n) \varphi_{n}(\alpha, r, x)\right) d x<S+O\left(\Phi\left(\Phi^{-1}(n) / n\right)\right) .
$$

Proof. By Lemma 2.1,

$$
\begin{aligned}
& \int_{-\pi}^{\pi} \Phi\left(n^{r} \Phi^{-1}(n) \varphi_{n}(\alpha, r, x)\right) d x \\
& <\frac{1}{2} \int_{-\pi}^{\pi} \Phi\left(\frac{2 \Phi^{-1}(n)}{\Gamma(r+1)} \varphi_{*}(\alpha, r, n x)\right) d x+\frac{1}{2} \int_{-\pi}^{\pi} \Phi\left(2 n^{r} \Phi^{-1}(n) \varepsilon_{n}(\alpha, r, x)\right) d x \\
& <c \int_{-\pi}^{\pi} \Phi\left(\Phi^{-1}(n) \varphi_{*}(\alpha, r, n x)\right) d x+O\left(\Phi\left(\Phi^{-1}(n) / n\right)\right) \\
& <c \int_{-n \pi}^{n \pi} \Phi\left(\varphi_{*}(\alpha, r, x)\right) d x+O\left(\Phi\left(\Phi^{-1}(n) / n\right)\right) .
\end{aligned}
$$


Now $\varphi_{*}(\alpha, r, x)=\cos (x+\alpha) h_{r+1}(x)-g_{r}(x) \sin (x+\alpha)$, and so

$$
\Phi\left(\left|\varphi_{*}(\alpha, r, x)\right|\right) \leqslant c\left[\Phi\left(h_{r+1}(x)\right)+\Phi\left(g_{r}(x)\right)\right]
$$

and

$$
\int_{-\infty}^{\infty} \Phi\left(\varphi_{*}(\alpha, r, x)\right) d x<c\left[\int_{-\infty}^{\infty} \Phi\left(h_{r+1}(x)\right) d x+\int_{-\infty}^{\infty} \Phi\left(g_{r}(x)\right) d x\right]<\infty,
$$

by Lemma 2.1D, which yields the conclusion of the lemma with

$$
S(\Phi, \alpha, r)=\int_{-\infty}^{\infty} \Phi\left(\varphi_{*}(\alpha, r, x)\right) d x .
$$

2.3 Lemma. Let $\Phi$ be a $\Delta_{2} N$-function and let $\delta>0, r>0$. Then there exists $a$ constant $C=C(r, \delta, \Phi)$ such that

$$
\int_{\delta<|x|<\pi} \Phi\left(n^{r} \Phi^{-1}(n) \varphi_{n}(\alpha, r, x)\right) d x=C \Phi\left(\Phi^{-1}(n) / n\right) .
$$

Proof. From the proof of Lemma 2.1, equation (5), we have that

$$
\begin{aligned}
\varphi_{n}(\alpha, r, x)= & \frac{1}{\Gamma(r+1)} \\
& \cdot \int_{0}^{1} \rho^{n}(\log 1 / \rho)^{r} \frac{(\cos ((n+1) x+\alpha)-\rho \cos (n x+\alpha))}{\rho^{2}-2 \rho \cos x+1} d \rho \\
< & \frac{1}{\Gamma(r+1)} \int_{0}^{1} \frac{2 \rho^{n-1}(\log 1 / \rho)^{r}}{4 \sin ^{2} x / 2} d \rho .
\end{aligned}
$$

For $|x|>\delta$,

$$
\int_{0}^{1} \frac{2 \rho^{n-1}(\log 1 / \rho)^{r}}{4 \sin ^{2} x / 2} d \rho<\frac{\pi^{2}}{4 \delta^{2}} \int_{0}^{1} \rho^{n-1}(\log 1 / \rho)^{r} d \rho=\frac{\pi^{2}}{4 \delta^{2}} \cdot \frac{\Gamma(r+1)}{n^{r+1}}
$$

so

$$
\begin{aligned}
\int_{\delta<|x|<\pi} \Phi\left(n^{r} \Phi^{-1}(n) \varphi_{n}(\alpha, r, x)\right) d x & <\int_{\delta<|x|<\pi} \Phi\left(\frac{\pi^{2}}{4 \delta^{2}} \cdot \frac{\Phi^{-1}(n)}{n}\right) d x \\
& <C \Phi\left(\Phi^{-1}(n) / n\right) .
\end{aligned}
$$

We define

$$
\varphi_{(0), 0}(x)=\sum_{k=1}^{\infty} k^{-1} \sin k x=\frac{\pi-x}{2}, \quad 0<x<2 \pi .
$$

Then, 


$$
\begin{aligned}
\varphi_{(r), 0}(x) & =\int D^{(r)}(x-t) \varphi_{(0), 0}(t) d t \\
& =\sum_{k=1}^{\infty} k^{-r-1} \cos \left(k x-\frac{r+1}{2} \pi\right)=\varphi_{0}\left(-\frac{r+1}{2} \pi, r, x\right) .
\end{aligned}
$$

We note that $S\left[\varphi_{(r), 0}\right]=\varphi_{(r), 0}, r \geqslant 0$, so that we shall write

$$
\begin{aligned}
\varphi_{(r), 0}-S_{n}\left(\varphi_{(r), 0)}\right. & =\varphi_{(r), n}(x)=\sum_{k=n+1}^{\infty} \frac{\cos (k x-(r+1) \pi / 2)}{k^{r+1}} \\
& =\int D^{(r)}(x-t) \varphi_{(0), n}(t) d t .
\end{aligned}
$$

2.4 Lemma. Let $\Phi$ be a $\Delta^{\prime} N$-function such that $\int_{|t|>1} \Phi\left(t^{-1}\right) d t<\infty$ and let $r>0$. Suppose $-\pi<x_{1}<x_{2}<\cdots<x_{m}<\pi$ and define

$$
f(x)=\sum_{k=1}^{m} \sigma_{k} \varphi(r), 0\left(x-x_{k}\right)
$$

where $\sigma=\left\{\sigma_{k}\right\}$ is a sequence of real numbers. If

$$
0<\delta<\min \left\{\pi+\frac{x_{1}-x_{m}}{2}, \frac{x_{2}-x_{1}}{2}, \ldots, \frac{x_{m}-x_{m-1}}{2}\right\},
$$

then

$$
\left\|f-S_{n}(f)\right\|_{\Phi}^{\prime}<\frac{2}{n^{r} \Phi^{-1}(n)} \cdot 1 / \Phi^{-1}\left(\frac{1}{\frac{S C_{\Phi}^{2}}{2} \sum_{j=1}^{m} \Phi\left(\sigma_{j}\right)+O_{m, \delta, \sigma}\left(\Phi\left(\frac{\Phi^{-1}(n)}{n}\right)\right)}\right)
$$

where $f(n)=O_{m, \delta, \sigma}(g(n))$ means $|f(n) / g(n)| \leqslant c$ where $c=c(m, \delta, \sigma)$.

Proof.

$$
\begin{aligned}
\frac{1}{2} \| n^{r} \Phi^{-1}(n) & \left(f-S_{n}(f)\right) \|_{\Phi}^{\prime} \\
& =\inf \left\{k: \int_{-\pi}^{\pi} \Phi\left(\frac{n^{r} \Phi^{-1}(n)}{2 k}\left(f-S_{n}(f)\right)\right) d x<1\right\} \\
& <\inf \left\{k: C_{\Phi} \Phi\left(\frac{1}{k}\right) \int_{-\pi}^{\pi} \Phi\left(\frac{n^{r} \Phi^{-1}(n)}{2}\left(f-S_{n}(f)\right)\right) d x<1\right\} .
\end{aligned}
$$

Let

$$
R=R(\Phi, n, f)=C_{\Phi} \int_{-\pi}^{\pi} \Phi\left(\frac{n^{r} \Phi^{-1}(n)\left(f-S_{n}(f)\right)}{2}\right) d x
$$

then 


$$
\left\|\frac{n^{r} \Phi^{-1}(n)}{2}\left(f-S_{n}(f)\right)\right\|_{\Phi}^{\prime}<\frac{1}{\Phi^{-1}(1 / R)} .
$$

Now we need only determine $R$.

Let $\chi_{j}$ denote the characteristic function of the set $\left(x_{j}-\delta, x_{j}+\delta\right), j=$ $2, \ldots, m-1$, of the set $\left(x_{1}-\delta, x_{1}+\delta\right) \cup\left(x_{1}-\delta+2 \pi, \pi\right)$ for $j=1$, and of the set $\left(x_{m}-\delta, x_{m}+\delta\right) \cup\left(-\pi, x_{m}+\delta-2 \pi\right)$ for $j=m$. Then

$$
\left(f-S_{n}(f)\right)(x)=\sum_{j=1}^{m} x_{j}(x) \sigma_{j} \varphi_{(r), n}\left(x-x_{j}\right)+\sum_{j=1}^{m}\left(1-x_{j}(x)\right) \sigma_{j} \varphi_{(r), n}\left(x-x_{j}\right)
$$

and

$$
\begin{aligned}
\frac{R}{C_{\Phi}}=\int_{-\pi}^{\pi} \Phi\left(\frac{n^{r} \Phi^{-1}(n)}{2} \sum_{j=1}^{\infty} \chi_{j}(x) \sigma_{j} \varphi_{(r), n}\left(x-x_{j}\right)\right. \\
\left.\quad+\frac{n^{r} \Phi^{-1}(n)}{2} \sum_{j=1}^{m}\left(1-x_{j}(x)\right) \sigma_{j} \varphi_{(r), n}\left(x-x_{j}\right)\right) d x \\
<\frac{1}{2} \int_{-\pi}^{\pi} \Phi\left(n^{r} \Phi^{-1}(n) \sum_{j=1}^{m} \chi_{j}(x) \sigma_{j} \varphi_{(r), n}\left(x-x_{j}\right)\right) d x \\
\quad+\frac{1}{2} \int_{-\pi}^{\pi} \Phi\left(n^{r} \Phi^{-1}(n) \sum_{j=1}^{m}\left(1-x_{j}(x)\right) \sigma_{j} \varphi_{(r), n}\left(x-x_{j}\right)\right) d x \\
=\frac{1}{2} \sum_{j=1}^{m} \int_{x_{j}-\delta}^{x+\delta} \Phi^{r}\left(n^{r} \Phi^{-1}(n) \sigma_{j} \varphi_{(r), n}\left(x-x_{j}\right)\right) d x \\
\quad+O_{m, \delta, \sigma}\left(\Phi\left(\Phi^{-1}(n) / n\right)\right),
\end{aligned}
$$

since, by Lemma 2.3 ,

$$
\int_{-\pi}^{\pi} \Phi\left(n^{r} \Phi^{-1}(n)\left(1-\chi_{j}(x)\right) \sigma_{j} \varphi_{(r), n}\left(x-x_{j}\right)\right) d x=O_{m, \delta, \sigma}\left(\Phi\left(\Phi^{-1}(n) / n\right)\right) .
$$

By Lemma.2.2,

$$
\begin{aligned}
\frac{1}{2} \int_{x_{j}-\delta}^{x_{j}+\delta} \Phi\left(n^{r} \Phi^{-1}(n) \sigma_{j} \varphi_{(r), n}\left(x-x_{j}\right)\right) d x \\
<\frac{C_{\Phi}}{2} \Phi\left(\sigma_{j}\right) \int_{x_{j}-\delta}^{x_{j}+\delta} \Phi\left(n^{r} \Phi^{-1}(n) \varphi_{(r), n}\left(x-x_{j}\right)\right) d x \\
<\left(C_{\Phi} / 2\right) \Phi\left(\sigma_{j}\right)\left(S+O\left(\Phi\left(\Phi^{-1}(n) / n\right)\right)\right.
\end{aligned}
$$

and we have, finally, 


$$
\begin{aligned}
\frac{R}{C_{\Phi}} & <\frac{C_{\Phi}}{2} \sum_{j=1}^{m} \Phi\left(\sigma_{j}\right)\left(S+O\left(\Phi\left(\frac{\Phi^{-1}(n)}{n}\right)\right)\right)+O_{m, \delta, \sigma}\left(\Phi\left(\frac{\Phi^{-1}(n)}{n}\right)\right) \\
& =\frac{C_{\Phi} S}{2} \sum_{j=1}^{m} \Phi\left(\sigma_{j}\right)+O_{m, \delta, \sigma}\left(\Phi\left(\frac{\Phi^{-1}(n)}{n}\right)\right) .
\end{aligned}
$$

Thus,

$$
\left\|f-S_{n}(f)\right\|_{\Phi}^{\prime}<\frac{2}{n^{r} \Phi^{-1}(n)} \cdot 1 / \Phi^{-1}\left(\frac{1}{\frac{C_{\Phi}^{2} S}{2} \sum_{j=1}^{m} \Phi\left(\sigma_{j}\right)+O_{m, \delta, \sigma}\left(\Phi\left(\frac{\Phi^{-1}(n)}{n}\right)\right)}\right]
$$

as desired.

2.5 Lemma. Let $\Phi$ be an $N$-function and $f \in \mathfrak{V}_{\Phi}^{*}$. Then there exists $a \neq 0$ such that

$$
\sup _{|h|<\delta} \int_{0}^{2 \pi} \Phi(a f(x+h)-a f(x)) d x \leqslant 3 \delta V_{\Phi}^{(2 \delta)}(a f)
$$

Proof. Since $f \in \mathcal{V}_{\Phi}^{*}$, there exist $k \neq 0$ such that $k f \in \mathcal{V}_{\Phi}$. If 1 is such a $k$, then $f \in \mathfrak{V}_{\Phi}$ and let $a=1$, otherwise let $a$ be any $k$ as above. Fix $\delta>0$ and take any $h \in(0, \delta]$. Let $n$ be the least integer greater than or equal to $2 \pi / h$, and let $t_{i}=i h, i=0,1, \ldots, n-1, t_{n}=2 \pi, t_{n+1}=2 \pi+h$. Further define

$$
\begin{gathered}
t_{i}^{\prime}= \begin{cases}t_{i}, & i=0,1, \ldots, n-1, n+1, \\
t_{n-1}+h, & i=n .\end{cases} \\
\int_{0}^{2 \pi} \Phi(a f(t+h)-a f(t)) d t=\sum_{i=1}^{n} \int_{t_{i-1}}^{t_{1}} \Phi(a f(x+h)-a f(t)) d t \\
\quad<\sum_{i=1}^{n}\left(t_{i}-t_{i-1}\right) \sup _{t \in\left[t_{i-1}, t_{i}\right]} \Phi(a f(t+h)-a(f(t))) \\
<h \sum_{i=1}^{n} \sup _{t \in\left[t_{i-1}, t_{i}\right]} \Phi(a f(t+h)-a f(t))
\end{gathered}
$$

Dividing this sum into two parts, we let $\Sigma_{0}$ denote the sum over even $i$, and let $\Sigma_{1}$ denote the sum over odd $i$. Then 


$$
\begin{aligned}
\int_{0}^{2 \pi} \Phi(a f(t+h)-a f(t)) d t< & h \Sigma_{0} \sup _{t \in\left[t_{i-1}, t_{i}\right]} \Phi(a f(t+h)-a f(t)) \\
& +h \Sigma_{1} \sup _{t \in\left[t_{i-1}, t_{i}\right]} \Phi(a f(t+h)-a f(t)) \\
< & h \Sigma_{0} \sup _{x, y \in\left[t_{i-1}^{\prime}, t_{i+1}^{\prime}\right]} \Phi(a f(x)-a f(y)) \\
& +h \Sigma_{1} \lim _{x, y \in\left[t_{i-1}^{\prime}, t_{i+1}^{\prime}\right]} \Phi(a f(x)-a f(y)) .
\end{aligned}
$$

If $2 \pi$ is an integral multiple of $h$ then there is no overlapping of the intervals in each summation. Otherwise, if $n$ is even, $\left[2 \pi-t_{n-2}^{\prime}, t_{n}^{\prime}-2 \pi\right] \cap$ $\left[0, t_{2}^{\prime}\right]=\left[0, t_{1}^{\prime}\right]$, so we let $\Sigma_{1}^{\prime}$ be the sum over all odd $i$, except $n-1$; if $n$ is odd then $\left[2 \pi-t_{n-1}^{\prime}, t_{n+1}^{\prime}-2 \pi\right] \cap\left[0, t_{2}^{\prime}\right]=\left[0, t_{1}^{\prime}\right]$, so let $\Sigma_{1}^{\prime}$ be the sum over all odd $i$ except $n$.

Fix $\varepsilon>0$. We may choose $x_{i}^{\prime}, y_{i}^{\prime} \in\left[t_{i-1}^{\prime}, t_{i+1}^{\prime}\right]$ for all $i$ so that

$$
\begin{aligned}
\Sigma_{0} \sup _{x, y \in\left[t_{i-1}^{\prime}, t_{i+1}^{\prime}\right]} \Phi(a f(x)-a f(y)) & <\Sigma_{0}\left[\Phi\left(a f\left(x_{i}^{\prime}\right)-a f\left(y_{i}^{\prime}\right)\right)+\varepsilon / n\right] \\
& <\Sigma_{0} \Phi\left(a f\left(x_{i}^{\prime}\right)-a f\left(y_{i}^{\prime}\right)\right)+\varepsilon, \\
\Sigma_{1}^{\prime} \sup _{x, y \in\left[t_{i-1}^{\prime}, t_{i+1}^{\prime}\right]} \Phi(a f(x)-a f(y)) & <\Sigma_{1}^{\prime}\left[\Phi\left(a f\left(x_{1}^{\prime}\right)-a f\left(y_{i}^{\prime}\right)\right)+\varepsilon / n\right] \\
& <\Sigma_{1}^{\prime} \Phi\left(a f\left(x_{i}^{\prime}\right)-a f\left(y_{i}^{\prime}\right)\right)+\varepsilon,
\end{aligned}
$$

and, $n$ even,

$$
\sup _{x, y \in\left[t_{n-2}^{\prime}, t_{n}^{\prime}\right]} \Phi(a f(x)-a f(y))<\Phi\left(a f\left(x_{n-1}^{\prime}\right)-a f\left(y_{n-1}^{\prime}\right)\right)+\varepsilon / n
$$

or, $n$ odd,

$$
\sup _{x, y \in\left[t_{n-1}^{\prime}, t_{n+1}^{\prime}\right]} \Phi(a f(x)-a f(y))<\Phi\left(a f\left(x_{n}^{\prime}\right)-a f\left(y_{n}^{\prime}\right)\right)+\varepsilon / n .
$$

Now, $\left|x_{i}^{\prime}-y_{i}^{\prime}\right| \leqslant 2 h, i=1, \ldots, n$, and so

$$
\begin{aligned}
\int_{0}^{2 \pi} \Phi(a f(x+h) & -a f(x)) d x<h\left(\Sigma_{0} \Phi\left(a f\left(x_{i}\right)-a f\left(y_{i}\right)\right)+\varepsilon\right) \\
& +h\left(\Sigma_{1}^{\prime} \Phi\left(a f\left(x_{i}^{\prime}\right)-a f\left(y_{i}^{\prime}\right)\right)+\varepsilon\right)+h\left(V_{\Phi}^{(2 h)}(a f)+\varepsilon / n\right) \\
< & 3 h\left(V_{\Phi}^{(2 h)}(a f)+\varepsilon\right) .
\end{aligned}
$$

Since $\varepsilon$ is arbitrary, we have

$$
\sup _{|h|<\delta} \int_{0}^{2 \pi} \Phi(a f(x+h)-a f(x)) d x<3 \delta V_{\Phi}^{(2 \delta)}(a f) .
$$

2.6 Lemma. Suppose $\Phi$ and $\Psi$ are $N$-functions, $\Psi$ is $\Delta_{2}$, and $\lim _{x \rightarrow 0} \Psi(x) / \Phi(x)=0$. Then $\mathfrak{V}_{\Phi} \subset W_{\Psi}^{*}=\left\{f \in \mathfrak{V}_{\Psi}: V_{\Psi}^{*}(f)=\sum_{j=1}^{\infty} \Psi\left(\sigma_{j}\right)\right.$ 
where $\left\{\sigma_{j}\right\}$ is the sequence of jumps of $\left.f\right\}$.

Proof. Let $\varepsilon>0$. Suppose $f \in \mathcal{V}_{\Phi},\left\{x_{k}\right\}$ its points of discontinuity and $\left\{\sigma_{k}\right\}$ the corresponding sequence of jumps of $f$.

Define

$$
g_{m}(x)=\sum_{k=1}^{m} \sigma_{k} \varphi_{(0), 0}\left(x-x_{k}\right)
$$

and let

$$
h_{m}(x)=f(x)-g_{m}(x) .
$$

Since $\lim _{x \rightarrow 0} \Psi(x) / \Phi(x)=0$, there exists $\lambda=\lambda(\varepsilon)$ such that, when $|x|<\lambda$,

$$
\Psi(x)<\varepsilon \Phi(x) / V_{\Phi}(f)+2 .
$$

Now $f \in \mathcal{V}_{\Phi}$ implies that $\Sigma \Phi\left(\sigma_{k}\right)<\infty$ which implies $\left|\sigma_{k}\right| \rightarrow 0$. Thus, there is an $N=N(\lambda, \varepsilon)$ such that $\Sigma_{N+1}^{\infty} \Psi\left(\sigma_{k}\right)<\Psi(\lambda / 4)$. We have that $h_{N}$ is continuous at $x_{1}, \ldots, x_{N}$ and that the oscillation of $h_{N}$ at $x \in[0,2 \pi]$ is less than $\lambda / 4$, so there exists an $\eta_{1}>0$ so that $\omega\left(\eta_{1}, h_{N}\right)<\lambda / 2$.

Next, since $g_{N}$ is a piecewise linear function, there exists $\eta_{2}$ so that $\left|g_{N}(\beta)-g_{N}(\alpha)\right|<\lambda / 2$ for $(\beta-\alpha)<\eta_{2}$ and $\alpha, \beta$ on the same interval of linearity. Let $\delta_{0}=\min \left(\eta_{1}, \eta_{2}\right)$.

$$
|f(\beta)-f(\alpha)| \leqslant\left|g_{N}(\beta)-g_{N}(\alpha)\right|+\left|h_{N}(\beta)-h_{N}(\alpha)\right|<\lambda
$$

whenever $|\beta-\alpha|<\delta_{0}$ and $\left\{x_{i}\right\}_{i=1}^{N} \cap[\alpha, \beta]=\varnothing$.

Let $\gamma$ be the minimum length of the intervals of linearity of $g_{N}$, and select $\delta_{1}<\min \left(\delta_{0}, \gamma\right)$ so that when

(3) $\alpha<x_{i}<\beta$ and $|\beta-\alpha|<\delta_{1}$,

$$
\Psi(f(\beta)-f(\alpha))<\Psi\left(\sigma_{i}\right)+\varepsilon /\left(N\left(V_{\Phi}(f)+2\right)\right), \quad i=1, \ldots, N,
$$

and when

(4) $x_{i}=\alpha$ or $x_{i}=\beta,|\beta-\alpha|<\delta_{1}$,

$$
\Psi(f(\beta)-f(\alpha))<\Psi\left(\sigma_{i} / 2\right)+\varepsilon /\left(N\left(V_{\Phi}(f)+2\right)\right) .
$$

We now choose a partition $\left\{y_{j}\right\}$ of $[0,2 \pi]$ with mesh less than $\delta_{1}$, so that $\left\{x_{i}\right\}_{i=1}^{N} \cap\left\{y_{j}\right\}=\varnothing$. Denote by $\Sigma_{a}$ the summation over all $j$ where $\left\{x_{i}\right\}_{i=1}^{N} \cap$ $\left[y_{j-1}, y_{j}\right]=\varnothing$, and by $\Sigma_{b}$ the summation over all $j$ where $\left\{x_{i}\right\}_{i=1}^{N} \cap\left[y_{j-1}\right.$, $\left.y_{j}\right] \neq \varnothing$. Then from (1) and (2),

$$
\begin{aligned}
\Sigma_{a} \Psi\left(f\left(y_{j}\right)-f\left(y_{j-1}\right)\right) & =\Sigma_{a} \frac{\Psi\left(f\left(y_{j}\right)-f\left(y_{j-1}\right)\right)}{\Phi\left(f\left(y_{j}\right)-f\left(y_{j-1}\right)\right)} \Phi\left(f\left(y_{j}\right)-f\left(y_{j-1}\right)\right) \\
& \leqslant \frac{\varepsilon}{V_{\Phi}(f)+2} \Sigma_{a} \Phi\left(f\left(y_{j}\right)-f\left(y_{j-1}\right)\right) .
\end{aligned}
$$

For $\Sigma_{b}$, we have, from (3), that, 


$$
\Sigma_{b} \Psi\left(f\left(y_{i}\right)-f\left(y_{j-1}\right)\right)<\sum_{i=1}^{N}\left(\Psi\left(\sigma_{i}\right)+\varepsilon /\left(N\left(V_{\Phi}(f)+2\right)\right)\right) .
$$

Hence,

$$
\begin{aligned}
\Sigma \Psi\left(f\left(y_{j}\right)-f\left(y_{j-1}\right)\right)< & \frac{\varepsilon}{V_{\Phi}(f)+2} \Sigma_{a} \Phi\left(f\left(y_{j}\right)-f\left(y_{j-1}\right)\right) \\
& +\frac{\varepsilon}{V_{\Phi}(f)+2}+\sum_{j=1}^{N} \Psi\left(\sigma_{i}\right) \\
& <\varepsilon+\sum_{i=1}^{\infty} \Psi\left(\sigma_{i}\right) .
\end{aligned}
$$

Now, on the other hand, suppose we choose a partition $\left\{z_{j}\right\}$ with mesh less than $\delta_{1}$ such that $\left\{x_{i}\right\}_{i=1}^{N} \cap\left\{z_{j}\right\} \neq \varnothing$. Let

$\Sigma_{a}$ denote the sum over all $j$ where $\left\{x_{i}\right\}_{i=1}^{N} \cap\left[z_{j-1}, z_{j}\right]=\varnothing$,

$\Sigma_{b}$ denote the sum over all $j$ where $\left\{z_{j-1}, z_{j}\right\} \cap\left\{x_{i}\right\}_{i=1}^{N} \neq \varnothing$ and

$\Sigma_{c}$ denote the sum over all $j$ where $\left(z_{j-1}, z_{j}\right) \cap\left\{x_{i}\right\}_{i=1}^{N} \neq \varnothing$.

Proceeding as before, $\Sigma_{a} \Psi\left(f\left(z_{j}\right)-f\left(z_{j-1}\right)\right)<\varepsilon V_{\Phi}(f) /\left(V_{\Phi}(f)+2\right)$. If we denote by $\Sigma_{b}^{\prime}$ the sum over all $i$ such that $x_{i}=z_{j}$ for some $i$ and $j$, then

$$
\begin{aligned}
\Sigma_{b} \Psi\left(f\left(z_{j}\right)-f\left(z_{j-1}\right)\right) & <2 \Sigma_{b}^{\prime}\left[\Psi\left(\sigma_{i} / 2\right)+\varepsilon /\left(N\left(V_{\Phi}(f)+2\right)\right)\right] \\
& <\Sigma_{b}^{\prime} \Psi\left(\sigma_{i}\right)+2 \varepsilon\left(\Sigma_{b}^{\prime}(1)\right) /\left(N\left(V_{\Phi}(f)+2\right)\right) .
\end{aligned}
$$

Letting $\Sigma_{c}^{\prime}$ denote the sum over all $i$ such that $x_{i} \in\left(z_{j-1}, z_{j}\right)$ for some $j$, we have

$$
\Sigma_{c} \Psi\left(f\left(z_{j}\right)-f\left(z_{j-1}\right)\right)<\Sigma_{c}^{\prime}\left[\Psi\left(\sigma_{i}\right)+\varepsilon /\left(N\left(V_{\Phi}(f)+2\right)\right)\right] .
$$

Thus

$$
\begin{aligned}
& \Sigma \Psi\left(f\left(z_{i}\right)-f\left(z_{i-1}\right)\right)=\Sigma_{a}+\Sigma_{b}+\Sigma_{c} \\
& \quad<\frac{\varepsilon V_{\Phi}(f)}{V_{\Phi}(f)+2}+\Sigma_{b}^{\prime} \frac{2 \varepsilon}{N\left(V_{\Phi}(f)+2\right)}+\Sigma_{c}^{\prime} \frac{\varepsilon}{N\left(V_{\Phi}(f)+2\right)}+\sum_{i=1}^{N} \Psi\left(\sigma_{i}\right) \\
& \quad<\sum_{i=1}^{\infty} \Psi\left(\sigma_{i}\right)+\varepsilon .
\end{aligned}
$$

Thus, for any partition $\left\{w_{j}\right\}$ of mesh less than $\delta_{1}$,

$$
\Sigma \Psi\left(f\left(w_{j}\right)-f\left(w_{j-1}\right)\right)<\sum_{j=1}^{\infty} \Psi\left(\sigma_{i}\right)+\varepsilon
$$

and

$$
V_{\Psi}^{*}(f) \leqslant \sum_{i=1}^{\infty}\left(\sigma_{i}\right)+\varepsilon
$$


Since $\varepsilon$ is arbitrary, and we also know that $V_{\Psi}^{*}(f) \geqslant \sum_{i=1}^{\infty} \Psi\left(\sigma_{i}\right)$ for all functions $f \in \mathfrak{V}_{\Psi}$, where $\left\{\sigma_{i}\right\}$ is the sequence of jumps of $f$, the desired result follows.

2.7 Lemma. Suppose $f^{(r)} \in L_{\Phi}^{*}, r \geqslant 0$, then $L_{\Phi}^{*}$ reflexive implies

$$
\left\|f-S_{n}(f)\right\|_{\Phi} \leqslant \frac{c}{n^{r}} \omega_{\Phi}\left(\frac{1}{n} ; f^{(r)}\right) .
$$

Proof. For $f \in L_{\Phi}^{*}$, Ryan [10] has shown $\left\|S_{n}(f)\right\|_{\Phi} \leqslant c\|f\|_{\Phi}$, where $c$ is independent of $n$ and $f$ if and only if $L_{\Phi}^{*}$ is reflexive. Suppose $T_{n}(x)$ is an $n$th degree trigonometric polynomial of best approximation to $f$ and define

$$
E_{n}(f)_{\Phi}=\left\|f(x)-T_{n}(x)\right\|_{\Phi}
$$

Reflexivity of $L_{\Phi}^{*}$ yields

$$
\left\|f-S_{n}(f)\right\|_{\Phi} \leqslant\left\|f-T_{n}\right\|_{\Phi}+\left\|S_{n}\left(T_{n}-f\right)\right\|_{\Phi} \leqslant(c+1) E_{n}(f)_{\Phi} .
$$

We now use a result which will be proved below:

For $f^{(r)} \in L_{\Phi}^{*}$, there exists $c=c(r, \Phi)$, such that

$$
E_{n}(f)_{\Phi} \leqslant \frac{c}{n^{r}} \omega_{\Phi}\left(\frac{1}{n} ; f^{(r)}\right) .
$$

Equations (1) and (*) combined yield the result.

To prove (*) we need the following definitions and results from Sz.-Nagy [7]

1. Suppose $\Sigma_{j m}=\left\{\sigma_{j}, \ldots, \sigma_{m-1}, \tau_{j}, \ldots, \tau_{m-1}\right\}$ and $g \in L^{1}, g(x) \sim$ $\sum_{k=j}^{\infty} \alpha_{k} \cos k x+\beta_{k} \sin k x$. Define a "regular approximation to $g(x)$ " by

$$
g_{m-1}\left(\sum_{j m} ; x\right)=\sum_{k=j}^{m-1} \sigma_{k}\left(\alpha_{k} \cos k x+\beta_{k} \sin k x\right)+\tau_{k}\left(\beta_{k} \cos k x-\alpha_{k} \sin k x\right) \text {. }
$$

2. Henceforth, $\lambda=\left\{\lambda_{k}\right\}_{k=1}^{\infty}$ denotes a three-fold monotone sequence converging to zero $\left(\lambda_{j}>0, \lambda_{j} \downarrow 0\right)$, for which $\Sigma\left(\lambda_{k} / k\right)<\infty$, and let $\delta$ be an arbitrary real number. If

define

$$
f(x) \sim \sum_{k=j}^{\infty} a_{k} \cos k x+b_{k} \sin k x,
$$

$$
\begin{aligned}
\left(T_{\lambda \delta}^{j} f\right)(x) & =\sum_{k=j}^{\infty} \lambda_{k}\left(a_{k} \cos \left(k x+\frac{1}{2} \delta \pi\right)+b_{k} \sin \left(k x+\frac{1}{2} \delta \pi\right)\right) \\
& =\int H_{\lambda \delta}^{j}(y) f(x+y) d y
\end{aligned}
$$

where

$$
H_{\lambda \delta}^{j}(y)=\sum_{k=j}^{\infty} \lambda_{k} \cos \left(k x-\frac{1}{2} \delta \pi\right)
$$


The series on the right in (2) is a Fourier series converging to an integrable function, $H_{\lambda \delta}^{j}$, and the series in (1) is a convergent sequence almost everywhere.

3. THEOREM. If $\delta=0$, there exists a set $\Sigma_{1 m}^{r}=\left\{\check{\sigma}_{1}, \ldots, \check{\sigma}_{m-1}\right.$, $\left.\check{\tau}_{1}, \ldots, \check{\tau}_{m-1}\right\}$ and a polynomial

$$
P_{\lambda 0}^{m-1}(x)=\sum_{k=1}^{m-1} \check{\xi}_{k} \cos k x+\check{\eta}_{k} \sin k x
$$

where for $1 \leqslant k \leqslant m-1, \check{\xi}_{k}=\lambda_{k}\left(1-\check{\sigma}_{k}\right), \check{\eta}_{k}=-\lambda_{k} \check{\tau}_{k}$, such that

$$
\int_{0}^{2 \pi}\left|H_{\lambda 0}^{m}(y)+P_{\lambda 0}^{m-1}(y)\right| d y=\frac{4}{\pi} \sum_{\nu=0}^{\infty}(-1)^{\nu} \frac{\lambda_{(2 \nu+1) m}}{2 \nu+1} .
$$

4. THEOREM. If $\delta=1$, there exists a set $\Sigma_{1 m}^{n}=\left\{\hat{\sigma}_{1}, \ldots, \hat{\sigma}_{m-1}\right.$, $\left.\hat{\tau}_{1}, \ldots, \hat{\tau}_{m-1}\right\}$ and a polynomial

$$
P_{\lambda I}^{m-1}(x)=\sum_{k=1}^{m-1} \hat{\xi}_{k} \cos k x+\hat{\eta}_{k} \sin k x
$$

where for $1 \leqslant k \leqslant m-1, \hat{\xi}_{k}=\lambda_{k} \hat{\tau}_{k}, \hat{\eta}_{k}=\lambda_{k}\left(1-\hat{\sigma}_{k}\right)$, such that

$$
\int_{0}^{2 \pi}\left|H_{\lambda 1}^{m}(y)+P_{\lambda 1}^{m-1}(y)\right| d y=\frac{4}{\pi} \sum_{\nu=0}^{\infty} \frac{\lambda_{(2 \nu+1) m}}{2 \nu+1} .
$$

5. THEOREM. For $\delta$ arbitrary there exists a polynomial $P_{\lambda \delta}^{m-1}(x)$ such that

$$
\begin{aligned}
\int_{0}^{2 \pi}\left|H_{\lambda \delta}^{m}(y)+P_{\lambda \delta}^{m-1}(y)\right| d y< & \frac{4}{\pi}\left|\cos \frac{1}{2} \delta \pi\right| \sum_{\nu=0}^{\infty}(-1)^{\nu} \frac{\lambda_{(2 \nu+1) m}}{2 \nu+1} \\
& +\frac{4}{\pi}\left|\sin \frac{1}{2} \delta \pi\right| \sum_{\nu=0}^{\infty} \frac{\lambda_{(2 \nu+1) m}}{2 \nu+1} .
\end{aligned}
$$

Note that $H_{\lambda \delta}^{m}(x)=\cos \frac{1}{2} \delta \pi H_{\lambda 0}^{m}(x)+\sin \frac{1}{2} \delta \pi H_{\lambda 1}^{m}(x)$, so choose $P_{\lambda \delta}^{m-1}(x)$ $=\cos \frac{1}{2} \delta \pi P_{\lambda 0}^{m-1}(x)+\sin \frac{1}{2} \delta \pi P_{\lambda 1}^{m-1}(x)$. Select

$$
\Sigma_{1 m}^{*}=\left\{\sigma_{1}^{*}, \ldots, \sigma_{m-1}^{*}, \tau_{1}^{*}, \ldots, \tau_{m-1}^{*}\right\}
$$

so that if

$$
P_{\lambda \delta}^{m-1}(x)=\sum_{k=1}^{m-1} \xi_{k}^{*} \cos k x+\eta_{k}^{*} \sin k x
$$

then

$$
\begin{aligned}
& \xi_{k}^{*}=\lambda_{k} \cos \frac{\delta \pi}{2}-\lambda_{k}\left(\sigma_{k}^{*} \cos \frac{1}{2} \delta \pi-\tau_{k}^{*} \sin \frac{1}{2} \delta \pi\right), \\
& \eta_{k}^{*}=\lambda_{k} \sin \frac{1}{2} \delta \pi-\lambda_{k}\left(\sigma_{k}^{*} \sin \frac{1}{2} \delta \pi+\tau_{k}^{*} \cos \frac{1}{2} \delta \pi\right) .
\end{aligned}
$$

6. If $f(x)=\int H_{\lambda \delta}^{1}(y) g(x+y) d y$, then 


$$
\left|f(x)-f_{m-1}\left(\Sigma_{1 m}^{*} ; x\right)\right|=\left|\int\left(H_{\lambda \delta}^{m}(y)+P_{\lambda \delta}^{m-1}(y)\right) g(x+y) d y\right| .
$$

7. If $\lambda=\left\{k^{-\alpha}\right\}_{k=1}^{\infty}$, for $\alpha>0$, then $\lambda$ is a three-fold monotone decreasing sequence, $k^{-\alpha} \downarrow 0$, and $\Sigma k^{-\alpha-1}<\infty$. Let $\delta=-\alpha$. Then by $1-6$, there exists $\Sigma_{1 m}^{*}$ so that

$$
\begin{aligned}
\int_{0}^{2 \pi}\left|H_{\lambda,(-\alpha)}^{m}(y)+P_{\lambda,(-\alpha)}^{m-1}(y)\right| d y & \\
< & \frac{4}{\pi}\left|\cos \frac{1}{2} \alpha \pi\right| \sum_{\nu=0}^{\infty} \frac{(-1)^{\nu}}{(2 \nu+1)((2 \nu+1) m)^{\alpha}} \\
& +\frac{4}{\pi}\left|\sin \frac{1}{2} \alpha \pi\right| \sum_{\nu=0}^{\infty} \frac{1}{(2 \nu+1)((2 \nu+1) m)^{\alpha}} \\
< & \frac{1}{m^{\alpha}}\left[\frac{4}{\pi}\left|\cos \frac{1}{2} \alpha \pi\right| \sum_{\nu=0}^{\infty}(-1)^{\nu}(2 \nu+1)^{-1-\alpha}\right. \\
\quad & \left.\quad+\frac{4}{\pi}\left|\sin \frac{1}{2} \alpha \pi\right| \sum_{\nu=0}^{\infty}(2 \nu+1)^{-1-\alpha}\right] \\
< & \frac{1}{m^{\alpha}} C_{\alpha} .
\end{aligned}
$$

We continue with the proof of (*). If $f, g$ are as in 6 , and $g \in L_{\Phi}$, then

$$
\begin{aligned}
& \left\|f(x)-f_{m-1}\left(\Sigma_{1 m}^{*} ; x\right)\right\|_{\Phi} \\
& =\sup \left\{\int_{0}^{2 \pi} v(x) \int_{0}^{2 \pi} g(x+y)\left(H_{\lambda \delta}^{m}(y)+P_{\lambda \delta}^{m-1}(y)\right) d y d x:\right. \\
& \left.\int \Phi^{*}(v(x)) d x<1\right\} \\
& \leqslant\|g\|_{\Phi} \int_{0}^{2 \pi}\left|H_{\lambda \delta}^{m}(y)+P_{\lambda \delta}^{m-1}(y)\right| d y .
\end{aligned}
$$

Since $T_{\lambda,(-\alpha)}^{1} g, \lambda=\left\{k^{-\alpha}\right\}$, is just the fractional integral of $g$ of order $\alpha$, then $g=f^{(\alpha)}$, and, by 7

Thus

$$
\left\|f(x)-f_{m-1}\left(\Sigma_{1}^{*} ; x\right)\right\|_{\Phi} \leqslant\left\|f^{(\alpha)}\right\|_{\Phi} C_{\alpha} m^{-\alpha}
$$

Next, define

$$
E_{m}(f)_{\Phi} \leqslant C_{\alpha} m^{-\alpha}\left\|f^{(\alpha)}\right\|_{\Phi}
$$

$$
f(h ; x)=\frac{1}{h} \int_{-h / 2}^{h / 2} f(x+t) d t,
$$


and for $g \in L_{\Phi}$,

$$
\begin{aligned}
\left\|(g(h ; x))^{\prime}\right\|_{\Phi} & =\frac{1}{h} \sup \left\{\int v(x)(g(x+h / 2)-g(x-h / 2)) d x:\right. \\
\left.\qquad \Phi^{*}(v(x)) d x<1\right\} & \\
& =\frac{1}{h}\|g(x+h / 2)-g(x-h / 2)\|_{\Phi}<\frac{1}{h} \omega_{\Phi}(h ; g),
\end{aligned}
$$

and

$$
\begin{aligned}
& \|g(x)-g(h ; x)\|_{\Phi}=\sup \left\{\int_{0}^{2 \pi} v(x)(g(x)-g(h ; x)) d x:\right. \\
& \left.\quad \int \Phi^{*}(v(x)) d x \leqslant 1\right\} \\
& \quad=\sup \left\{\int_{0}^{2 \pi} v(x) \frac{1}{h} \int_{-h / 2}^{h / 2}(g(x)-g(x+t)) d t d x: \int \Phi^{*}(v(x)) d x<1\right\} \\
& \leqslant h^{-1} \int_{-h / 2}^{h / 2}\|g(x)-g(x+t)\|_{\Phi} d t \\
& <\omega_{\Phi}(h / 2 ; g) .
\end{aligned}
$$

Noting that $(f(x)-f(h ; x))^{(\alpha)}=f^{(\alpha)}-(f(h ; x))^{(\alpha)}=f^{(\alpha)}-f^{(\alpha)}(h ; x)$ almost everywhere, we have

$$
\begin{aligned}
E_{n}(f)_{\Phi} & \leqslant E_{n}(f-f(h ; \cdot))_{\Phi}+E_{n}(f(h ; \cdot))_{\Phi} \\
& <\frac{c_{\alpha}}{n^{\alpha}}\left\|f^{(\alpha)}-(f(h ; \cdot))^{(\alpha)}\right\|_{\Phi}+\frac{c_{\alpha+1}}{n^{\alpha+1}}\left\|(f(h ; \cdot))^{(\alpha+1)}\right\|_{\Phi} \\
& =\frac{c_{\alpha}}{n^{\alpha}}\left\|f^{(\alpha)}-f^{(\alpha)}(h ; \cdot)\right\|_{\Phi}+\frac{c_{\alpha+1}}{n^{\alpha+1}}\left\|\left(f^{(\alpha)}(h ; \cdot)\right)\right\|_{\Phi} \\
& <\frac{c_{\alpha}}{n^{\alpha}} \omega_{\Phi}\left(h / 2 ; f^{(\alpha)}\right)+\frac{c_{\alpha+1}}{n^{\alpha+1}} \cdot h^{-1} \omega_{\Phi}\left(h ; f^{(\alpha)}\right) .
\end{aligned}
$$

Now, letting $h=1 / h$, we have

$$
E_{n}(f)_{\Phi} \leqslant\left(c_{\alpha}+c_{\alpha+1}\right) n^{-\alpha} \omega_{\Phi}\left(n^{-1} ; f^{(\alpha)}\right)
$$

which is (*) when $\alpha=r$.

2.8 Lemma. Suppose $f \in \mathcal{V}_{\Phi}^{(r)}, r>0, \Phi$ an $N$-function. If $\Psi$ is a $\Delta^{\prime}$ $N$-function and $L_{\Phi}^{*}$ is reflexive then 
$\left\|f-S_{n}(f)\right\|_{\Psi}^{\prime}$

$$
<\frac{c}{n^{r} \Psi^{-1}(n)}\left[1 /\left(\Psi^{-1}\left(\frac{1}{c V_{\Phi}^{(2 / n)}\left(f^{(r)}\right)}\right) \Psi^{-1}\left(\frac{1}{\gamma\left(\Psi, \Phi, f^{(r)}, n^{-1}\right)}\right)\right)\right]
$$

where

$$
\gamma(\Psi, \Phi, g, h)=\sup \left\{\frac{\Psi(g(x+t)-g(x))}{\Phi(g(x+t)-g(x))}: x \in[0,2 \pi],|t| \leqslant h\right\} .
$$

Proof. Suppose $g(x) \in \mathfrak{T}_{\Phi}$. Then using Lemma 2.5,

$$
\begin{aligned}
& \|g(x+h)-g(x)\|_{\Psi}^{\prime}=\inf \left\{k: \int \Psi\left(\frac{g(x+h)-g(x)}{k}\right) d x<1\right\} \\
& \quad<\inf \left\{k: c \Psi(1 / k) \int \frac{\Psi(g(x+h)-g(x))}{\Phi(g(x+h)-g(x))} \cdot \Phi(g(x+h)-g(x)) d x<1\right\} \\
& \quad<\inf \left\{k: c \Psi(1 / k) \gamma(\Psi, \Phi, g, h) \int \Phi(g(x+h)-g(x)) d x<1\right\} \\
& \quad<\inf \left\{k: c \Psi(1 / k) \gamma(\Psi, \Phi, g, h) 3 h V_{\Phi}^{(2 h)}(g)<1\right\} .
\end{aligned}
$$

Thus

$$
\begin{aligned}
& \|g(x+h)-g(x)\|_{\Psi}^{\prime} \\
& \quad<1 /\left(\Psi^{-1}\left(\frac{1}{h}\right) \Psi^{-1}\left(\frac{1}{\gamma(\Psi, \Phi, g, h)}\right) \Psi^{-1}\left(\frac{c}{V_{\Phi}^{(2 h)}}(g)\right)\right) .
\end{aligned}
$$

By Lemma 2.7

$$
\begin{aligned}
\| f- & S_{n}(f) \|_{\Psi}<\frac{c_{r}^{\Psi}}{n^{r}} \omega_{\Psi}\left(\frac{1}{n} ; f^{(r)}\right) \\
& <\frac{c_{r}}{n^{r} \Psi^{-1}(n)} \cdot \frac{1}{\Psi^{-1}\left(c / V_{\Phi}^{(2 / n)}\left(f^{(r)}\right)\right)} \cdot \frac{1}{\Psi^{-1}\left(1 / \gamma\left(\Psi, \Phi, f^{(r)}, 1 / n\right)\right)} .
\end{aligned}
$$

We now return to the proof of the main theorem of this section.

Proof. Let

$$
u_{m}(x)=f^{(r)}(x)-\sum_{j=1}^{m} \sigma_{j} \varphi_{(0), 0}\left(x-x_{j}\right) .
$$

The oscillation of $u_{m}(x)$ at $x$ goes to zero uniformly in $x$ as $m \rightarrow \infty$ since $\Sigma \Phi\left(\sigma_{j}\right)<\infty$. We let $\gamma$ be as in Lemma 2.8 and select $\varepsilon>0$. Then there exists 
an $m_{0}$ and $\delta_{0}>0$ such that for all $m \geqslant m_{0}, \delta \leqslant \delta_{0}$,

$$
\gamma\left(\Psi, \Phi, u_{m} / 2, \delta\right)<1 / \Psi(1 / \varepsilon)
$$

since $\omega\left(u_{m} / 2, t\right) \rightarrow 0$ as $m \rightarrow \infty$ and $t \rightarrow 0$. Consider

$$
g(x)=\sum_{j=1}^{m_{0}} \sigma_{j} \varphi_{(r), 0}\left(x-x_{j}\right)
$$

and let $h(x)=f(x)-g(x)$. Then $h^{(r)}(x)=u_{m_{0}}(x)$. Now

$$
\left\|f-S_{n}(f)\right\|_{\Psi}^{\prime} \leqslant\left\|g-S_{n}(g)\right\|_{\Psi}^{\prime}+2\left\|h / 2-S_{n}(h / 2)\right\|_{\Psi}^{\prime} .
$$

Applying Lemma 2.8 and Lemma 2.4 with $\delta^{\prime}$ chosen as in that lemma,

$$
\begin{aligned}
\left\|f-S_{n}(f)\right\|_{\Psi}< & \frac{2}{n^{r} \Psi^{-1}(n)} \cdot\left[1 / \Psi^{-1}\left(\frac{1}{\frac{C_{\Psi}^{2} S}{2} \sum_{j=1}^{m_{0}} \Psi\left(\sigma_{j}\right)+O_{m_{0,8}, \sigma}\left(\Psi\left(\frac{\Psi^{-1}(n)}{n}\right)\right)}\right)\right] \\
& +\frac{c}{n^{r} \Psi^{-1}(n)} \frac{1}{\Psi^{-1}\left(c / V_{\Phi}^{(2 / n)}\left(h^{(r)} / 2\right)\right)} \cdot \frac{1}{\Psi^{-1}\left(1 / \gamma\left(\Psi, \Phi, h^{(r)} / 2,1 / n\right)\right)} .
\end{aligned}
$$

Now, select $n>1 / \delta_{0}$, which implies

$$
\gamma\left(\Psi, \Phi, \frac{u_{m_{0}}}{2}, \frac{1}{n}\right)<\frac{1}{\Psi(1 / \varepsilon)}
$$

and, therefore,

$$
\varepsilon>1 / \Psi^{-1}\left(1 / \gamma\left(\Psi, \Phi, h^{r}\right) / 2,1 / n\right)
$$

Thus,

$$
\begin{aligned}
\left\|f-S_{n}(f)\right\|_{\Psi}^{\prime}< & \frac{2}{n^{r} \Psi^{-1}(n)} \cdot\left[1 / \Psi^{-1}\left(\frac{1}{\frac{C_{\Psi}^{2} S}{2} \sum_{j=1}^{\infty} \Psi\left(\sigma_{j}\right)+O_{m_{0,8}, 0}\left(\Psi\left(\frac{\Psi^{-1}(n)}{n}\right)\right)}\right)\right] \\
& +\frac{c \varepsilon}{n^{r} \Psi^{-1}(n) \Psi^{-1}\left(c / V_{\phi}^{(2 / n)}\left(h^{(r)} / 2\right)\right)} .
\end{aligned}
$$

Now, suppose $\left\{\sigma_{i}\right\}_{i=1}^{\infty} \neq \varnothing$. Since $\Psi\left(\Psi^{-1}(n) / n\right) \rightarrow 0$ as $n \rightarrow \infty$, we have

$$
\begin{gathered}
\varlimsup_{n \rightarrow \infty}\left[\Psi^{-1}\left[1 /\left[\frac{C_{\Psi}^{2} S}{2} \sum_{j=1}^{\infty} \Psi\left(\sigma_{j}\right)+O_{m_{0}, \delta^{\prime}, \sigma}\left(\Psi\left(\frac{\Psi^{-1}(n)}{n}\right)\right)\right)\right]\right]^{-1} \\
=\left[\Psi^{-1}\left[1 /\left(\frac{C_{\Psi}^{2} S}{2} \sum_{j=1}^{\infty} \Psi\left(\sigma_{j}\right)\right)\right]\right]^{-1}
\end{gathered}
$$


and

$$
\varlimsup_{n \rightarrow \infty} \frac{c \varepsilon}{\Psi^{-1}\left(c / V_{\Phi}^{(2 / n)}\left(h^{(r)} / 2\right)\right)}=\frac{c \varepsilon}{\Psi^{-1}\left(c / V_{\Phi}^{*}\left(h^{(r)} / 2\right)\right)} .
$$

Since we know that

$$
V_{\Phi}^{*}\left(g^{(r)}\right)=\sum_{j=1}^{m_{0}} \Phi\left(\sigma_{j}\right)<V_{\Phi}^{*}\left(f^{(r)}\right)
$$

and $\left(f^{(r)}-g^{(r)}\right) / 2=h^{(r)} / 2$, we have

$$
V_{\Phi}^{*}\left(h^{(r)} / 2\right)<\frac{1}{2} V_{\Phi}^{*}\left(f^{(r)}\right)+\frac{1}{2} V_{\Phi}^{*}\left(g^{(r)}\right)<V_{\Phi}^{*}\left(f^{(r)}\right) .
$$

Hence,

$$
\begin{aligned}
\varlimsup_{n \rightarrow \infty} n^{r} \Psi^{-1}(n) \| f & -S_{n}(f) \|_{\Psi}^{\prime} \\
& <1 / \Psi^{-1}\left[\frac{1}{\frac{C_{\Psi}^{2} S}{2} \sum_{j=1}^{\infty} \Psi\left(\sigma_{j}\right)}\right]+\frac{c \varepsilon}{\Psi^{-1}\left(c / V_{\Phi}^{*}(f)\right)}
\end{aligned}
$$

where the right-hand side has no dependence on $m_{0}$. Since $\varepsilon$ is arbitrary, we have

$$
\varlimsup_{n \rightarrow \infty} n^{r} \Psi^{-1}(n)\left\|f-S_{n}(f)\right\|_{\Psi}^{\prime}<1 / \Psi^{-1}\left(\frac{1}{\frac{C_{\Psi}^{2} S}{2} \sum_{j=1}^{\infty} \Psi\left(\sigma_{j}\right)}\right) .
$$

If $f^{(r)}$ is continuous, we note that $g \equiv 0$ and (*) is replaced by

$$
\begin{aligned}
n^{r} \Psi^{-1}(n) & \left\|f-S_{n}(f)\right\|_{\Psi}^{\prime} \\
& <\frac{c}{\Psi^{-1}\left(c / V_{\Phi}^{(2 / n)}\left(f^{(r)}\right)\right)} \cdot 1 / \Psi^{-1}\left(\frac{1}{\gamma\left(\Psi, \Phi, f^{(r)}, 1 / n\right)}\right) \\
& =o(1) \text { as } n \rightarrow \infty .
\end{aligned}
$$

\section{BiBLIOGRAPHY}

1. N. Bari, Trigonometric series, Vols. I, II, Fizmatgiz, Moscow, 1961; English transl., Macmillan, New York, 1974. MR 23 \#A3411; 30 \# 1347.

2. B. I. Golubov, Continuous functions of bounded p-variation, Mat. Zametki 1 (1967), 305-312 = Math. Notes 1 (1967), 203-207. MR 35 \#2063.

3. - On functions of bounded p-variation, Izv. Akad. Nauk SSSR Ser. Mat. 32 (1968), 837-858 = Math. USSR Izv. 2 (1968), 799-819. MR 38 \#3392. 
4. M. A. Krasnosel'skii and Ja. B. Rutickii, Convex functions and Orlicz spaces, GITTL, Moscow, 1958; English transl., Noordhoff, Groningen, 1961. MR 21 \# 5144; 23 \#A4016.

5. E. R. Love, A generalization of absolute continuity, J. London Math. Soc. 26 (1951), 1-13. MR 12, 599.

6. J. Musielak and W. Orlicz, On generalized variations. I, Studia Math. 18 (1959), 11-41. MR 21 \#3524.

7. B. Sz.-Nagy, Über gewisse Extremalfragen bei transformierfen trigonometrischen Entwicklungen. I. Periodisches Fall, Berichten Math.-Phys. K1. Akad. Wiss. Leipzig 90 (1938), $103-134$.

8. S. M. Nikol'skii, Fourier series of functions having derivative of bounded variation, Izv. Akad. Nauk SSSR Ser. Mat. 13 (1949), 513-532. (Russian) MR 11, 348.

9. V. T. Pinkevič, Sur l'ordre du reste de la série de Fourier des fonctions derivable au sens de Weyl, Izv. Akad. Nauk SSSR Ser. Mat. 4 (1940), 521-528. (Russian) MR 2, 279.

10. R. Ryan, Conjugate functions in Orlicz spaces, Pacific J. Math. 13 (1963), 1371-1377. MR 28 \#420.

11. R. Salem, Essais sur les séries trigonométriques, Thèse présentée à la Faculté des Sciences de l'Université de Paris, Hermann, Paris, 1940. MR 2, 93, 419.

12. N. Wiener, The quadratic variation of a function and its Fourier coefficients, J. Math. and Phys. 3 (1924), 72-94.

13. L. C. Young, An inequality of the Hölder type connected with the Stieltjes integration, Acta Math. 67 (1936), 251-282.

14. A. Zygmund, Trignometrical series, Vols. I, II, 2nd rev. ed., Cambridge Univ. Press, New York, 1968. MR 38 \#4882.

Department of Computer Science, College of Engineering, University of Utah, Salt LAKE CitY, UTAH 84112

Current address: Departments of Computer Science and Mathematics, University of Utah, Salt Lake City, Utah 84112 